\title{
Three-Dimensional Target Localization and Cramér-Rao Bound for Two-Dimensional OFDM-MIMO Radar
}

\author{
Xingxing Li, Dangwei Wang, and Xiaoyan Ma \\ Air Force Early Warning Academy, Wuhan 430019, China \\ Correspondence should be addressed to Xingxing Li; li_x_xing@163.com
}

Received 14 June 2016; Revised 9 January 2017; Accepted 9 February 2017; Published 8 March 2017

Academic Editor: Angelo Liseno

Copyright (C) 2017 Xingxing Li et al. This is an open access article distributed under the Creative Commons Attribution License, which permits unrestricted use, distribution, and reproduction in any medium, provided the original work is properly cited.

Target localization using a frequency diversity multiple-input multiple-output (MIMO) system is one of the hottest research directions in the radar society. In this paper, three-dimensional (3D) target localization is considered for two-dimensional MIMO radar with orthogonal frequency division multiplexing linear frequency modulated (OFDM-LFM) waveforms. To realize joint estimation for range and angle in azimuth and elevation, the range-angle-dependent beam pattern with high range resolution is produced by the OFDM-LFM waveform. Then, the 3D target localization proposal is presented and the corresponding closed-form expressions of Cramér-Rao bound (CRB) are derived. Furthermore, for mitigating the coupling of angle and range and further improving the estimation precision, a CRB optimization method is proposed. Different from the existing methods of FDA-based radar, the proposed method can provide higher range estimation because of multiple transmitted frequency bands. Numerical simulation results are provided to demonstrate the effectiveness of the proposed approach and its improved performance of target localization.

\section{Introduction}

In the last few years, frequency diversity technology applied to multiple-input multiple-output (MIMO) systems has become one of the most popular research directions in the radar society. MIMO radar with the emerging technology provides additional degrees of freedom (DOFs) in range domain and produces the range-angle-dependent beam pattern to suppress range ambiguous clutter and interferences, which can enhance the received signal-to-interference-plus-noise ratio (SINR) and improve moving target detection performance [1-7].

In general, the existing MIMO radar with frequency diversity can be generally classified into two categories. The first type is MIMO radar with frequency diverse array (FDA). The radar referred to as FDA-MIMO radar employs a slight frequency increment across the elements so that the rangeangle-dependent beam pattern is achieved [5]. Range-angle dependency of the beam pattern allows the radar system to focus the transmit energy in a desired range-angle region [8]. The techniques of range-angle localization of targets by two pulses and subarray-based FDA radar are proposed in [9] and [10], respectively. The unambiguous method for angle and range estimation with a priori range estimate is proposed and the corresponding range and angle Cramér-Rao bounds (CRBs) are derived in [6]. Additionally, the CRBs of direction, range, and velocity with FDA array are derived in [11].

The second type is Frequency Diverse MIMO (FDMIMO) radar. Unlike the FDA-MIMO radar, the frequency interval between the adjacent array is large enough for the MIMO radar so that the spectrums of transmitted signals are disjoint $[12,13]$. As a result, the orthogonality of the transmitted signals is maintained and the waveform and frequency diversity can be achieved simultaneously. The phase difference of the received wave in each channel is determined by the array spatial structure, frequency increment, and the round-trip propagation time delay. The frequency increments across the subarrays add the frequency diversity information and the extra DOFs to the range bin containing the interested target in the receiver, which can acquire the range-angledependent beam pattern and some additional useful features. For example, the FD-MIMO radar can overcome grating lobe problem in distributed apertures MIMO systems [14], provide improved detection performance [1], and produce a larger virtual array aperture than the FDA [15]. 
Considering the useful advantages, three-dimensional (3D) target localization and its estimation performance are investigated for the FD-MIMO radar in this paper. For this purpose, the echo signal model of the MIMO radar with orthogonal frequency division multiplexing linear frequency modulated (OFDM-LFM) waveforms-termed OFDMMIMO radar-is presented first. Following this, the estimation approach is proposed for 3D target localization of our OFDM-MIMO radar and the corresponding closed-form expressions for the CRB on target's 3D parameters estimation are derived. Varying from the existing FDA-based radar for target localization, our radar employs two-dimensional (2D) array and transmits OFDM-LFM waveforms. Furthermore, $3 \mathrm{D}$ target parameters including range and angles in azimuth and elevation are jointly estimated, unlike the $2 \mathrm{D}$ parameters estimated in $[6,9,10,16]$. Moreover, since the range-angledependent beam pattern with high range resolution is produced by our OFDM-MIMO radar due to the OFDMLFM waveforms with large adjacent frequency interval, the range estimation precision can be higher than one of the existing FDA radar. The main contributions of this paper are as follows.

(1) The OFDM-MIMO radar with two-dimensional (2D) phased array and OFDM-LFM waveforms are used to jointly estimate $3 \mathrm{D}$ target parameters including range and angles in azimuth and elevation, which is different from the existing FDA-MIMO radar for 2D target localization in $[6,9,10,16]$. The proposed estimation approach for $3 \mathrm{D}$ target parameters utilizes the extra range bin DOFs associated with OFDMLFM waveforms to estimate the range parameter. The extra DOFs improve the range resolution of the range-angledependent beam pattern. Furthermore, the proposed estimation approach avoids a priori range estimate information and range compensation required in [6] and can exploit larger virtual array apertures for angle estimation which is not achieved in [10].

(2) The CRBs associated with 3D target parameters estimation are derived and the closed-form expressions of the CRBs are presented, which provides insight into the estimation performance of 3D target localization. Furthermore, a CRB optimization proposal based on frequency coding design is proposed, aiming to minimize the CRBs of the target's $3 \mathrm{D}$ parameters estimation.

The remainder of this paper is organized as follows. In Section 2, the echo signal model of the OFDM-MIMO radar is developed for $3 \mathrm{D}$ target localization. In Section 3, the estimation approach is presented for 3D target localization of our OFDM-MIMO radar. In Section 4, the closed-form expressions of the CRBs associated with 3D target parameters are derived and the CRBs are further optimized. Numerical examples are presented in Section 5. Finally, conclusions are given in Section 6.

\section{Echo Signal Model of Our OFDM-MIMO Radar}

As depicted in Figure 1, the case of $2 \mathrm{D}$ transmit and receive arrays is considered, where the arrays are placed in the $x-y$ plane of a three-dimensional coordinate system. The

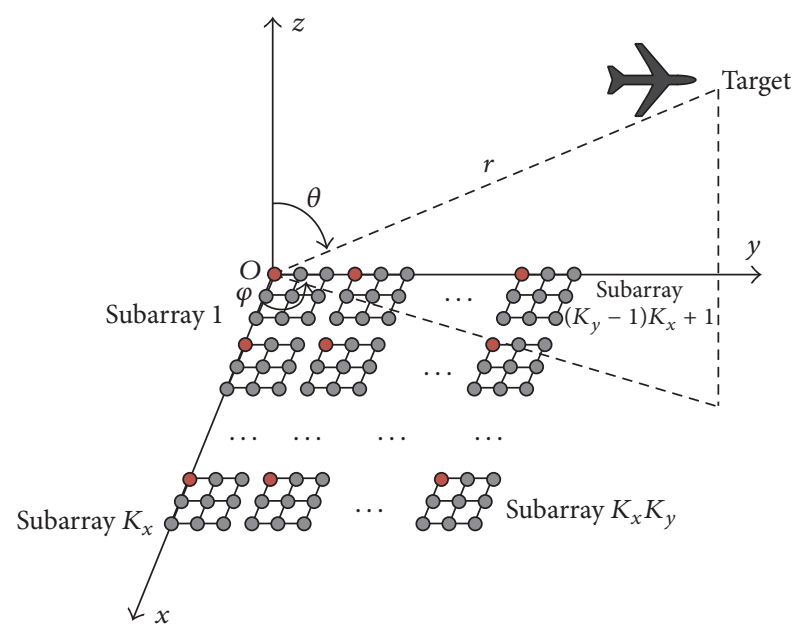

FIGURE 1: Geometry architecture of our OFDM-MIMO radar.

nonoverlapping subarrays considered in this paper are independent of each other, which can be steered in one or more directions with great agility. The $M$ elements are organized into $K=K_{x} \times K_{y}$ uniform rectangular subarrays, where $K_{x}$ and $K_{y}$ represent the number of subarrays in each row and column, respectively. Furthermore, each subarray contains $P=P_{x} \times P_{y}$ elements, where $P_{x}$ and $P_{y}$ represent the number of antennas in each row and column, respectively. The interspacing of the adjacent antenna elements at each row and column is represented by $d_{x}$ and $d_{y}$, respectively. Consequently, our OFDM-MIMO radar contains $K$ subarrays. Furthermore, allow all elements in each subarray to transmit a coherent OFDM-LFM waveform and receive the echoes from the transmitted waveforms of $K$ subarrays. The $k$ th transmit subarray OFDM-LFM waveform can be expressed as

$$
\phi_{k}(t)=\operatorname{rect}\left(\frac{t}{T}\right) \exp \left(j 2 \pi f_{k} t+j \pi \mu t^{2}\right)
$$

where $T$ denotes the pulse width; $\mu$ represents chirp rate; $B=\mu T$ denotes the bandwidth; $f_{k}$ is the carrier frequency of the $k$ th transmitted waveform which is given by

$$
f_{k}=f_{c}+(k-1) \Delta f, \quad k=1, \ldots, K,
$$

where $f_{c}$ denotes the reference carrier frequency and $\Delta f$ denotes the frequency interval between two adjacent transmitted waveforms.

For convenience, the three-dimensional coordinate system is transformed into spherical coordinates with $\theta$ and $\varphi$, which denote the elevation and azimuth, respectively. Without loss of generality, the first element of the first subarray is taken as the reference point (origin of the coordinate system $O x y z)$. Then, the $P \times 1$ transmit steering vector of the $k$ th subarray can be expressed as

$$
\begin{aligned}
\mathbf{C}_{k}(\theta, \varphi) & =\mathbf{a}_{k}(\theta, \varphi) A_{k}(\theta, \varphi) \\
& =\operatorname{vec}\left(\mathbf{u}_{k}(\theta, \varphi) \mathbf{v}_{k}^{T}(\theta, \varphi)\right) A_{k}(\theta, \varphi) \\
& =\left(\mathbf{v}_{k}(\theta, \varphi) \otimes \mathbf{u}_{k}(\theta, \varphi)\right) A_{k}(\theta, \varphi),
\end{aligned}
$$


where $A_{k}(\theta, \varphi)=\exp \left(j 2 \pi f_{k} \tau_{k}\right) ; \tau_{k}$ is the propagation time delay between the reference point (point $O$ ) and the first antenna of the $k$ th subarray; $\operatorname{vec}(\cdot)$ represents the operator that stacks the column of a matrix in one column vector; and $\otimes$ stands for the Kronecker product. $\mathbf{u}(\theta, \varphi) \in C^{P_{x} \times 1}$ and $\mathbf{v}(\theta, \varphi) \in C^{P_{y} \times 1}$ are given by

$$
\begin{aligned}
\mathbf{u}_{k} & (\theta, \varphi) \\
\quad= & {\left[1, e^{j 2 \pi f_{k} d_{x} \sin \theta \cos \varphi / c}, \ldots, e^{j 2 \pi f_{k}\left(P_{x}-1\right) d_{x} \sin \theta \cos \varphi / c}\right]^{T} } \\
\mathbf{v}_{k} & (\theta, \varphi) \\
\quad= & {\left[1, e^{j 2 \pi f_{k} d_{y} \sin \theta \sin \varphi / c}, \ldots, e^{j 2 \pi f_{k}\left(P_{y}-1\right) d_{y} \sin \theta \sin \varphi / c}\right]^{T}, }
\end{aligned}
$$

where $c$ denotes the wave propagation speed. Furthermore, let a target be located at a specific far field with the angle $(\theta, \varphi)$ and range $r$, where $r$ represents the distance between the reference point and the target. Under the point target assumption, the echo signals reflected by the hypothetical target and received at the reference point will have the following form [17]:

$$
s(t)=\sqrt{\frac{E}{K}} \alpha \sum_{k=1}^{K} \mathbf{w}_{k}^{H} \mathbf{a}_{k}(\theta, \varphi) A_{k}(\theta, \varphi) \phi_{k}\left(t-\frac{2 r}{c}\right),
$$

where $\alpha$ is the target reflection coefficient and $\mathbf{w}_{k}$ denotes the $P \times 1$ transmit beamformer weight vector in the $k$ th subarray. It is worth noting that the transmit power of the $k$ th subarray is $E / K$, where $E$ is the total transmit power. Steering all the subarrays in the same spatial angle $(\theta, \varphi)$, the nonadaptive transmit beamformer weight vectors can be expressed as

$$
\mathbf{w}_{k}=\frac{\mathbf{a}_{k}(\theta, \varphi)}{\left\|\mathbf{a}_{k}(\theta, \varphi)\right\|},
$$

where $\|\cdot\|$ denotes the $l_{2}$ norms. Then, (5) can be rewritten as

$$
s_{r}(t)=\sqrt{\frac{E}{K}} \alpha \mathbf{A}^{T}(\theta, \varphi) \phi\left(t-\frac{2 r}{c}\right),
$$

where $\phi(t)=\left[\phi_{1}(t), \phi_{2}(t), \ldots, \phi_{K}(t)\right]^{T}$ is the $K \times 1$ vector of the OFDM-LFM waveforms.

\section{Proposed Estimation Approach for 3D Target Localization}

3.1. Range Coarse Estimation. Three-dimensional target localization can be interpreted as computing the range, angles in azimuth, and elevation of a target in a given coordinate system. For this propose, $K$ matched filters are used in each element to separate $K$ echo signals from the linear combinations of the $K$ transmitted waveforms and then form the beam pattern with $P K \times K$ separated echo signals to estimate the target's parameters. In this subsection, 3D target localization of our OFDM-MIMO radar is considered in this way.

The received echo of the element in $m$ th row and $n$th column can be written as

$$
x_{m, n}=b_{m, n} s_{r}(t) \text {, }
$$

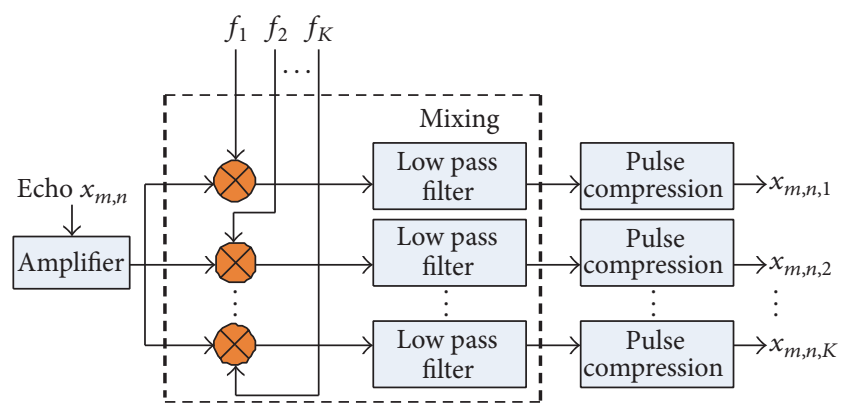

FIGURE 2: Signal processing diagram for each element.

where $b_{m, n}=e^{j 2 \pi f_{b}(m-1) d_{x} \sin \theta \cos \varphi / c} e^{j 2 \pi f_{b}(n-1) d_{y} \sin \theta \sin \varphi / c}$ is the element of $\mathbf{b}(\theta, \varphi)$ in $m$ th row and $n$th column; the steering vector of received arrays $\mathbf{b}(\theta, \varphi)$ has the following form:

$$
\mathbf{b}(\theta, \varphi)=\mathbf{b}_{x}(\theta, \varphi) \mathbf{b}_{y}^{T}(\theta, \varphi),
$$

where

$$
\begin{gathered}
\mathbf{b}_{x}(\theta, \varphi)=\left[1, e^{j 2 \pi f_{b} d_{x} \sin \theta \cos \varphi / c}, \ldots,\right. \\
\left.e^{j 2 \pi f_{b}\left(P_{x} K_{x}-1\right) d_{x} \sin \theta \cos \varphi / c}\right]^{T} \\
\mathbf{b}_{y}(\theta, \varphi)=\left[1, e^{j 2 \pi f_{b} d_{y} \sin \theta \sin \varphi / c}, \ldots,\right. \\
\left.e^{j 2 \pi f_{b}\left(P_{y} K_{y}-1\right) d_{y} \sin \theta \sin \varphi / c}\right]^{T},
\end{gathered}
$$

where $f_{b}=f_{c}+B(K-1) / 2$. By matched filtering $x_{m, n}$, as shown in Figure 2, the output signals can be arranged into the following $K \times 1$ data vector:

$$
\mathbf{y}_{m, n}=\left[x_{m, n, 1}, \ldots, x_{m, n, k} \ldots, x_{m, n, K}\right]^{T},
$$

where $x_{m, n, k}$ is the output of the $k$ th channel which can be expressed as [18]

$$
\begin{aligned}
x_{m, n, k}= & \sqrt{\frac{E}{K}} \sqrt{B T} \alpha b_{m, n} A_{k}(\theta, \varphi) \\
& \cdot \operatorname{rect}\left(\frac{t^{\prime}}{T}\right) \frac{\sin \pi B t^{\prime}}{\pi B t^{\prime}} \exp \left(-j 2 \pi f_{k} \frac{2 r}{c}\right),
\end{aligned}
$$

where $t^{\prime}=t-2 r / c$. In fact, the true range value (true time delay) can be represented as

$$
r=r_{a}+\Delta r
$$

where $r_{a}$ and $\Delta r$ are the integral multiple and the fraction of the range resolution cell, respectively. The coarse range estimate value $r_{a}$ can be obtained with the range bin number and bin size using the matched filtering algorithms in the time domain based on (12). The coarse estimation $r_{a}$ can be written as

$$
r_{a}=N_{a} \cdot \frac{c}{2 B},
$$

where $N_{a}$ denotes the range bin index of the target. The fine range estimation $\Delta r$ will be discussed in Section 3.2. 
3.2. 3D Target Localization. Digitally sampling the output signal in (12) and ignoring the reduction (less than $3 \mathrm{~dB}$ ) in the outputs signal amplitude due to the sampling, (12) becomes

$$
x_{m, n, k}=\beta b_{m, n} A_{k}(\theta, \varphi) \exp \left(-j 2 \pi f_{k} \frac{2 r}{c}\right) \text {, }
$$

where $\beta=\sqrt{M / K} \sqrt{B T} \alpha$. The steering vector with range dependence brought by the frequency diverse channels can be defined as

$$
\begin{gathered}
\mathbf{R}(r)=\left[1, \exp \left(-j 2 \pi \Delta f \frac{2 r}{c}\right), \ldots,\right. \\
\left.\exp \left(-j 2 \pi(K-1) \Delta f \frac{2 r}{c}\right)\right]^{T} .
\end{gathered}
$$

After an inspection of the expression above, one can observe that the phase of the received wave in each channel is different for the same propagation distance, which offer the extra range bin DOFs in the range domain. Utilizing the phase difference in each channel, we can estimate $\Delta r$ accurately in range beam domain. Besides, by observing the vector $\mathbf{R}(r)$ carefully, we can find that it is a periodic function relative to $r$, and furthermore the period is $c /(2 \Delta f)$. It is easy to verify that $\mathbf{R}(r+$ $c / 2 \Delta f)=\mathbf{R}(r)$. In this work, the case of $\Delta f=B$ is employed. Therefore, we can reach the interesting result that shows the period associated with range is equal to range bin size; that is, it equals the range resolution ratio of each transmitted signal $\phi_{k}(t)$. So, the range dependence compensation in [6] is not necessary for this paper. Based on (14),

$$
\mathbf{R}(r)=\mathbf{R}\left(r-r_{a}\right)=\mathbf{R}(\Delta r) .
$$

Thus, $\Delta r$ can be estimated directly from the beamforming output peaks in range beam domain. Our range estimation approach can be depicted as two steps: the coarse range estimation $r_{a}$ is obtained by the matched filtering algorithms in the time domain, and then the fine estimation $\Delta r$ is obtained by phase differences [see also (16)] among the frequency diversity channels in beam domain. Consequently, the true range estimate is given by $\widehat{r}=r_{a}+\Delta \widehat{r}$.

Next, we discuss the estimation method of $(\theta, \varphi, \Delta r)$. For clarity, (15) can be rewritten as

$$
x_{m, n, k}=\beta b_{m, n} A_{k}(\theta, \varphi) R_{k}(r) .
$$

The term $\exp \left(-j 2 \pi f_{c} \cdot 2 r / c\right)$ is lumped into $\beta$ in (18) and does not appear in the following processing for reasons of simplicity. $R_{k}(r)$ is the $k$ th element of vector $\mathbf{R}(r)$. Hence, (11) becomes

$$
\begin{aligned}
\mathbf{y}_{m, n} & =\left[x_{m, n, 1}, \ldots, x_{m, n, k}, \ldots, x_{m, n, K}\right]^{T} \\
= & {\left[\beta b_{m, n} A_{1}(\theta, \varphi) R_{1}(r), \beta b_{m, n} A_{2}(\theta, \varphi)\right.} \\
& \left.\cdot R_{2}(r), \ldots, \beta b_{m, n} A_{K}(\theta, \varphi) R_{K}(r)\right]^{T}=\beta b_{m, n}[\mathbf{A}(\theta, \varphi) \\
& \circ \mathbf{R}(r)]=\beta b_{m, n}\left[\mathbf{A}(\theta, \varphi) \circ \mathbf{R}\left(r-r_{a}\right)\right] \\
& =\beta b_{m, n}[\mathbf{A}(\theta, \varphi) \circ \mathbf{R}(\Delta r)],
\end{aligned}
$$

where $\mathbf{A}(\theta, \varphi)=\left[1, A_{2}(\theta, \varphi), \ldots, A_{K}(\theta, \varphi)\right]^{T}$ and $\circ$ is the Hadamard product. Finally, the following virtual data vector can be formed:

$$
\begin{aligned}
\mathbf{z} & =\left[\begin{array}{cccc}
\mathbf{y}_{1,1} & \mathbf{y}_{1,2} & \vdots & \mathbf{y}_{1, K_{y} P_{y}} \\
\mathbf{y}_{2,1} & \mathbf{y}_{2,2} & \vdots & \mathbf{y}_{2, K_{y} P_{y}} \\
\vdots & \vdots & \mathbf{y}_{m, n} & \vdots \\
\mathbf{y}_{K_{x} P_{x}, 1} & \mathbf{y}_{K_{x} P_{x}, 2} & \vdots & \mathbf{y}_{K_{x} P_{x}, K_{y} P_{y}}
\end{array}\right] \\
& =\beta \mathbf{b}(\theta, \varphi) \otimes[\mathbf{A}(\theta, \varphi) \circ \mathbf{R}(\Delta r)] \\
& =\beta \mathbf{b}(\theta, \varphi) \otimes \mathbf{a}(\theta, \varphi, r),
\end{aligned}
$$

where $\mathbf{a}(\theta, \varphi, r)=\mathbf{A}(\theta, \varphi) \circ \mathbf{R}(\Delta r)$ is the equivalent transmit steering vector. One can observe that the vector $\mathbf{a}(\theta, \varphi, r)$ is not only angle-dependent but also range-dependent. When rewriting the matrix $\mathbf{b}(\theta, \varphi)$ in one column vector,

$$
\mathbf{b}_{\mathrm{vec}}(\theta, \varphi)=\operatorname{vec}(\mathbf{b}(\theta, \varphi))=\mathbf{b}_{y}(\theta, \varphi) \otimes \mathbf{b}_{x}(\theta, \varphi) .
$$

Then, $\mathbf{z}$ can be rewritten in one column vector

$$
\begin{aligned}
\mathbf{z}_{\mathrm{vec}} & =\operatorname{vec}(\mathbf{z})=\beta \mathbf{b}_{\mathrm{vec}}(\theta, \varphi) \otimes \mathbf{a}(\theta, \varphi, r) \\
& =\beta \boldsymbol{\mu}(\theta, \varphi, r) .
\end{aligned}
$$

Suppose that the target is observed in the background noise. In that case, (22) can be rewritten as

$$
\mathbf{z}_{\mathrm{vec}}=\beta \boldsymbol{\mu}(\theta, \varphi, r)+\mathbf{n},
$$

where $K P K \times 1$ noise vector $\mathbf{n}$ is supposed to be circularly Gaussian distributed with zero mean and covariance $\mathbf{C}_{n}=$ $\sigma_{n}^{2} \mathbf{I}_{K P K}$ and $\mathbf{I}_{K P K}$ is the identity matrix of order KPK. Here, the simplifying assumption is made where the signal and the noise are independent. Then we obtain the covariance matrix of $\mathbf{z}_{\mathrm{vec}}$ :

$$
\mathbf{R}_{\mathbf{z}_{\mathrm{vec}}}=E\left[\mathbf{z}_{\mathrm{vec}} \mathbf{z}_{\mathrm{vec}}^{H}\right]
$$

where $E[\cdot]$ is the mathematical expectation; thus, we obtain the eigendecomposition:

$$
\mathbf{R}_{\mathbf{z}_{\mathrm{vec}}}=\mathbf{E}_{s} \Lambda_{s} \mathbf{E}_{s}^{H}+\mathbf{E}_{n} \Lambda_{n} \mathbf{E}_{n}^{H},
$$

where $\mathbf{E}_{s}$ and $\mathbf{E}_{n}$ are the signal- and noise-subspace matrices, respectively. The diagonal matrices $\Lambda_{s}$ and $\Lambda_{n}$ are the corresponding eigenvalues. The MUSIC cost function can be expressed as

$$
P_{\text {MUSIC }}(\theta, \varphi, \Delta r)=\frac{\boldsymbol{\mu}(\theta, \varphi, \Delta r)^{H} \boldsymbol{\mu}(\theta, \varphi, \Delta r)}{\boldsymbol{\mu}(\theta, \varphi, \Delta r)^{H} \mathbf{E}_{n} \mathbf{E}_{n}^{H} \boldsymbol{\mu}(\theta, \varphi, \Delta r)}
$$

Then the parameters with respect to $(\theta, \varphi, \Delta r)$ can be estimated by the peaks of (26):

$$
(\widehat{\theta}, \widehat{\varphi}, \Delta \widehat{r})=\arg \max _{(\theta, \varphi, \Delta r)}\left|P_{\text {MUSIC }}(\theta, \varphi, \Delta r)\right| .
$$


3.3. Characteristics Analysis. The characteristics of the $3 \mathrm{D}$ target localization for our OFDM radar can be summarized as follows. (1) Parameter identifiability capability: the maximum number of targets that can be uniquely identified is $P K^{2}-1$ which can be observed from (20). (2) Resolution and measurement accuracy: our system can exploit the effect aperture of virtual array to obtain better angle estimation performance and use the extra range bin DOFs to achieve higher range estimation precision. (3) Computational complexity: the computational burden of the standard MUSIC is $O\left(N^{2}(l+2)+J(N+1)(N-l)\right)$ [19], where $l$ is the number of sources, $J$ denotes the total sample points of spatial spectral over $[-\pi / 2, \pi / 2]$, and $N$ is the dimensions of the source covariance matrix. Thus, the computational cost of our method is $O\left(P^{2} K^{4}(l+2)+J_{r} J_{\theta} J_{\varphi}\left(P K^{2}+1\right)\left(P K^{2}-l\right)\right)$, where $J_{\theta}$ and $J_{\varphi}$ denote the total sample points of spatial spectral over $[-\pi / 2, \pi / 2]$ and $J_{r}$ denotes the total sample points of spatial spectral over $[-c / 4 B, c / 4 B]$.

\section{Performance Analysis of Our FD-MIMO Radar}

4.1. Derivation of the CRBs. The CRB plays an extraordinary role in parameter estimation because it is usually used as a benchmark to assess unbiased estimators. To investigate the performance lower bound of our OFDM-MIMO radar, the $\mathrm{CRB}$ expressions for angle and range estimation are derived. The deterministic parameter vector of date model (23) is

$$
\psi=\left[\theta, \varphi, r, \beta_{R}, \beta_{I}\right]^{T}
$$

where $\beta_{R}=\operatorname{Re}\{\beta\}, \beta_{I}=\operatorname{Im}\{\beta\}, \operatorname{Re}\{\cdot\}$, and $\operatorname{Im}\{\cdot\}$ are the real and imaginary parts, respectively. The corresponding Fisher information matrix (FIM) [20] for $\psi$ can be computed as

$$
\mathbf{J}=2 L \cdot \operatorname{Re}\left\{\frac{\partial(\beta \boldsymbol{\mu}(\psi))^{H}}{\partial \psi} C_{n}^{-1} \frac{\partial(\beta \boldsymbol{\mu}(\psi))}{\partial \psi}\right\}
$$

where $C_{n}=\sigma^{2} \mathbf{I}$ and $L$ is the number of snapshots. Three auxiliary vectors are defined as $\boldsymbol{\mu}_{\theta}=\partial \boldsymbol{\mu}(\psi) / \partial \theta, \boldsymbol{\mu}_{\varphi}=$ $\partial \boldsymbol{\mu}(\psi) / \partial \varphi$, and $\boldsymbol{\mu}_{r}=\partial \boldsymbol{\mu}(\psi) / \partial r$. Subsequently, the auxiliary vectors are computed:

$$
\begin{aligned}
\boldsymbol{\mu}_{\theta} & \\
= & \frac{\partial\left[\mathbf{b}_{y}(\theta, \varphi) \otimes \mathbf{b}_{x}(\theta, \varphi) \otimes\left[\mathbf{A}(\theta, \varphi) \circ \mathbf{R}\left(r-r_{a}\right)\right]\right]}{\partial \theta} \\
= & \frac{\partial \mathbf{b}_{y}(\theta, \varphi)}{\partial \theta} \otimes \mathbf{b}_{x}(\theta, \varphi) \otimes\left[\mathbf{A}(\theta, \varphi) \circ \mathbf{R}\left(r-r_{a}\right)\right] \\
& +\mathbf{b}_{y}(\theta, \varphi) \otimes \frac{\partial \mathbf{b}_{x}(\theta, \varphi)}{\partial \theta} \\
& \otimes\left[\mathbf{A}(\theta, \varphi) \circ \mathbf{R}\left(r-r_{a}\right)\right]+\mathbf{b}_{y}(\theta, \varphi) \otimes \mathbf{b}_{x}(\theta, \varphi) \\
& \otimes\left[\frac{\partial \mathbf{A}(\theta, \varphi)}{\partial \theta} \circ \mathbf{R}\left(r-r_{a}\right)\right]
\end{aligned}
$$

$$
\begin{aligned}
= & {\left[\gamma_{2} \mathbf{D}_{y} \mathbf{b}_{y}(\theta, \varphi)\right] \otimes \mathbf{b}_{x}(\theta, \varphi) } \\
& \otimes\left[\mathbf{A}(\theta, \varphi) \circ \mathbf{R}\left(r-r_{a}\right)\right]+\mathbf{b}_{y}(\theta, \varphi) \\
& \otimes\left[\gamma_{1} \mathbf{D}_{x} \mathbf{b}_{x}(\theta, \varphi)\right] \otimes\left[\mathbf{A}(\theta, \varphi) \circ \mathbf{R}\left(r-r_{a}\right)\right] \\
& +\mathbf{b}_{y}(\theta, \varphi) \otimes \mathbf{b}_{x}(\theta, \varphi) \\
& \otimes\left[\gamma_{3} \circ \mathbf{A}(\theta, \varphi) \circ \mathbf{R}\left(r-r_{a}\right)\right],
\end{aligned}
$$

where $\partial \mathbf{b}_{x}(\theta, \varphi) / \partial \theta=\gamma_{1} \mathbf{D}_{x} \mathbf{b}_{x}(\theta, \varphi), \quad \partial \mathbf{b}_{y}(\theta, \varphi) / \partial \theta=$ $\gamma_{2} \mathbf{D}_{y} \mathbf{b}_{y}(\theta, \varphi)$, and $\partial \mathbf{A}(\theta, \varphi) / \partial \theta=\gamma_{3} \circ \mathbf{A}(\theta, \varphi)$.

The following vectors are introduced:

$$
\begin{aligned}
\gamma_{1} & =\frac{j 2 \pi f_{b} d_{x} \cos \theta \cos \varphi}{c} \\
\mathbf{D}_{x} & =\operatorname{diag}\left[0,1, \ldots, P_{x} K_{x}-1\right] \\
\gamma_{2} & =\frac{j 2 \pi f_{b} d_{y} \cos \theta \sin \varphi}{c} \\
\mathbf{D}_{y} & =\operatorname{diag}\left[0,1, \ldots, P_{y} K_{y}-1\right] \\
\gamma_{3} & =\left[\gamma_{3}(1), \gamma_{3}(2), \ldots, \gamma_{3}(K)\right]^{T} \\
\gamma_{3}(k) & =\frac{\partial\left(j 2 \pi f_{k} \tau_{k}\right)}{\partial \theta} .
\end{aligned}
$$

Similarly, we can obtain the other auxiliary vectors:

$$
\begin{aligned}
\boldsymbol{\mu}_{\varphi} & \\
= & \frac{\partial\left[\mathbf{b}_{y}(\theta, \varphi) \otimes \mathbf{b}_{x}(\theta, \varphi) \otimes\left[\mathbf{A}(\theta, \varphi) \circ \mathbf{R}\left(r-r_{a}\right)\right]\right]}{\partial \varphi} \\
= & {\left[\gamma_{5} \mathbf{D}_{y} \mathbf{b}_{y}(\theta, \varphi)\right] \otimes \mathbf{b}_{x}(\theta, \varphi) } \\
& \otimes\left[\mathbf{A}(\theta, \varphi) \circ \mathbf{R}\left(r-r_{a}\right)\right]+\mathbf{b}_{y}(\theta, \varphi) \\
& \otimes\left[\gamma_{4} \mathbf{D}_{x} \mathbf{b}_{x}(\theta, \varphi)\right] \otimes\left[\mathbf{A}(\theta, \varphi) \circ \mathbf{R}\left(r-r_{a}\right)\right] \\
& +\mathbf{b}_{y}(\theta, \varphi) \otimes \mathbf{b}_{x}(\theta, \varphi) \\
& \otimes\left[\gamma_{6} \circ \mathbf{A}(\theta, \varphi) \circ \mathbf{R}\left(r-r_{a}\right)\right] \\
\boldsymbol{\mu}_{r} & \frac{\partial\left[\mathbf{b}_{y}(\theta, \varphi) \otimes \mathbf{b}_{x}(\theta, \varphi) \otimes\left[\mathbf{A}(\theta, \varphi) \circ \mathbf{R}\left(r-r_{a}\right)\right]\right]}{\partial r} \\
= & \mathbf{b}_{y}(\theta, \varphi) \otimes \mathbf{b}_{x}(\theta, \varphi) \\
& \otimes\left[\gamma_{r} \circ \mathbf{A}(\theta, \varphi) \circ \mathbf{R}\left(r-r_{a}\right)\right],
\end{aligned}
$$

where

$$
\begin{aligned}
\gamma_{4} & =\frac{-j 2 \pi f_{b} d_{x} \sin \theta \sin \varphi}{c} \\
\gamma_{5} & =\frac{j 2 \pi f_{b} d_{y} \sin \theta \cos \varphi}{c}
\end{aligned}
$$




$$
\begin{aligned}
\gamma_{6} & =\left[\gamma_{6}(1), \gamma_{6}(2), \ldots, \gamma_{6}(K)\right]^{T} \\
\gamma_{6}(k) & =\frac{\partial\left(j 2 \pi f_{k} \tau_{k}\right)}{\partial \varphi} \\
\gamma_{r} & =-j 4 \pi \Delta f \cdot \frac{\operatorname{diag}(0,1,2, \ldots, K-1)}{c} .
\end{aligned}
$$

In the outcome, the closed-form CRBs for angle $(\theta, \varphi)$ and range $r$ are given by

$$
\begin{aligned}
& D_{\theta}=\frac{1}{2 L \operatorname{LNR} \operatorname{det}\left(\mathbf{Q}_{0}\right)}\left(\left\|\boldsymbol{\mu}_{\varphi}\right\|^{2}-\frac{\left|\boldsymbol{\mu}_{\varphi}^{H} \boldsymbol{\mu}\right|^{2}}{\|\boldsymbol{\mu}\|^{2}}\right)\left(\left\|\boldsymbol{\mu}_{r}\right\|^{2}\right. \\
& \left.-\frac{\left|\boldsymbol{\mu}_{r}^{H} \boldsymbol{\mu}\right|^{2}}{\|\boldsymbol{\mu}\|^{2}}\right)-\frac{1}{2 L \mathrm{SNR} \operatorname{det}\left(\mathbf{Q}_{0}\right)}\left(\operatorname{Re}\left\{\boldsymbol{\mu}_{\varphi}^{H} \boldsymbol{\mu}_{r}\right\}\right. \\
& \left.-\frac{\operatorname{Re}\left\{\boldsymbol{\mu}^{H} \boldsymbol{\mu}_{\varphi} \boldsymbol{\mu}_{r}^{H} \boldsymbol{\mu}\right\}}{\|\boldsymbol{\mu}\|^{2}}\right)^{2} \\
& D_{\varphi}=\frac{1}{2 L S N R \operatorname{det}\left(\mathbf{Q}_{0}\right)}\left(\left\|\boldsymbol{\mu}_{\theta}\right\|^{2}-\frac{\left|\boldsymbol{\mu}_{\theta}^{H} \boldsymbol{\mu}\right|^{2}}{\|\boldsymbol{\mu}\|^{2}}\right)\left(\left\|\boldsymbol{\mu}_{r}\right\|^{2}\right. \\
& \left.-\frac{\left|\boldsymbol{\mu}_{r}^{H} \boldsymbol{\mu}\right|^{2}}{\|\boldsymbol{\mu}\|^{2}}\right)-\frac{1}{2 L \mathrm{SNR} \operatorname{det}\left(\mathbf{Q}_{0}\right)}\left(\operatorname{Re}\left\{\boldsymbol{\mu}_{\theta}^{H} \boldsymbol{\mu}_{r}\right\}\right. \\
& \left.-\frac{\operatorname{Re}\left\{\boldsymbol{\mu}^{H} \boldsymbol{\mu}_{\theta} \boldsymbol{\mu}_{r}^{H} \boldsymbol{\mu}\right\}}{\|\boldsymbol{\mu}\|^{2}}\right)^{2} \\
& D_{r}=\frac{1}{2 L S N R \operatorname{det}\left(\mathbf{Q}_{0}\right)}\left(\left\|\boldsymbol{\mu}_{\theta}\right\|^{2}-\frac{\left|\boldsymbol{\mu}_{\theta}^{H} \boldsymbol{\mu}\right|^{2}}{\|\boldsymbol{\mu}\|^{2}}\right)\left(\left\|\boldsymbol{\mu}_{\varphi}\right\|^{2}\right. \\
& \left.-\frac{\left|\boldsymbol{\mu}_{\varphi}^{H} \boldsymbol{\mu}\right|^{2}}{\|\boldsymbol{\mu}\|^{2}}\right)-\frac{1}{2 L \mathrm{SNR} \operatorname{det}\left(\mathbf{Q}_{0}\right)}\left(\operatorname{Re}\left\{\boldsymbol{\mu}_{\theta}^{H} \boldsymbol{\mu}_{\varphi}\right\}\right. \\
& \left.-\frac{\operatorname{Re}\left\{\boldsymbol{\mu}^{H} \boldsymbol{\mu}_{\theta} \boldsymbol{\mu}_{\varphi}^{H} \boldsymbol{\mu}\right\}}{\|\boldsymbol{\mu}\|^{2}}\right)^{2} .
\end{aligned}
$$

The derivation of (34) is provided in the Appendix. It can be observed that the CRBs are relevant to the array aperture, subarrays arrangement, frequency increment, the angle of the target, and frequency coding sequence. After an inspection of the data model (20), we can make the following qualitative conclusions: (1) more subarrays mean better performance for elevation and azimuth angle estimation due to the improvement in the angular resolution (a larger aperture) and for range estimation owing to the higher range resolution. (2) As the frequency increment increases, more accurate range estimations can be achieved owing to larger equivalent bandwidth. (3) The elevation and azimuth angle estimation accuracy depends on the array aperture, and the range is mainly related to the frequency increment under the same SNR.
4.2. CRBs Optimization. As the FDA with sequential frequency coding has range-angle coupling, the range and angle CRBs will be degraded when estimating the range and angle jointly [11]. In this paper, the range-angle is also coupled in the transmitter which can be observed via the equivalent transmit steering vectors $\mathbf{a}(\theta, \varphi, r)$ [see also (20)]. In fact, the nonlinear frequency increments can also be applied to FDA and different frequency shifts will result in different beam patterns, which provide an additional DOF to control the range-angledependence beamforming [4]. In other words, the nonsequenced frequency coding offers a way to alleviate the coupling of range and angle, and we can obtain improved parameters estimation precision by employing an appropriate frequency coding. To achieve better performance of parameters estimation, the CRBs for range and angle can be minimized by optimizing the frequency coding. The optimization problem can be modeled as

$$
\begin{gathered}
\min _{\mathbf{k}} \operatorname{trace}\left(\mathbf{Q}_{0}^{-1}\right) \\
\text { s.t } \quad \mathbf{k} \in \mathbb{C},
\end{gathered}
$$

where $\mathbb{C}$ denotes all the permutations of $K$ integers $([0,1, \ldots$, $K-1])$. The model of (35) can be solved using the genetic algorithm (GA); thus, we obtain the optimized frequency coding sequence $f_{k}=f_{c}+\mathbf{k} \Delta f$.

\section{Simulation and Verification}

In this section, several simulations are conducted to verify the theoretical analysis and the effectiveness of the proposed OFDM-MIMO radar in terms of 3D target localization. Our OFDM-MIMO radar uses the following simulation parameters: $f_{0}=10 \mathrm{GHz}, \Delta f=B=1 \mathrm{MHz}, T=10 \mu \mathrm{s}$, and $d_{x}=d_{y}=c /\left(2 f_{\max }\right)$, where $f_{\max }$ is the maximal transmit frequency and the number of snapshots $L=100$. Furthermore, we suppose a target of interest is located at direction $(\theta, \varphi)=$ $\left(60^{\circ}, 60^{\circ}\right)$ and range $r=15.105 \mathrm{~km}$ unless otherwise is specified.

5.1. 3D Target Localization and Influence of Gain-Phase Errors of Arrays. We validate the performance of target localization with our OFDM-MIMO radar in this section. We have $N_{a}=$ 100 due to $r=15.105 \mathrm{~km}$; that is, the target is located in the 100th range bin and $\Delta r=105 \mathrm{~m}$. Consider $P=P_{x} \times P_{y}=$ $2 \times 2$ and $K=K_{x} \times K_{y}=2 \times 4$. Figures 3, 4, and 5 show the root mean square errors (RMSEs) of angle and range versus SNR, respectively, which is obtained by 500 Monte Carlo simulation runs. It is noted from Figures 3, 4, and 5 that the range estimating accuracy is much higher than the range resolution $(150 \mathrm{~m})$; therefore the proposed mothed can provide the superresolution in range due to the additional DOFs in range. Besides, the accuracy can be improved further with the increase of SNR in our system while this cannot be realized in phased array radar since the range resolution is determined by the bandwidth of the transmitted signal. Moreover, the proposed OFDM-MIMO radar gives a satisfactory estimation performance for the angle and range, and the RMSEs of the angle and range can achieve the theoretical CRB when the SNR is higher than $-10 \mathrm{~dB}$. 


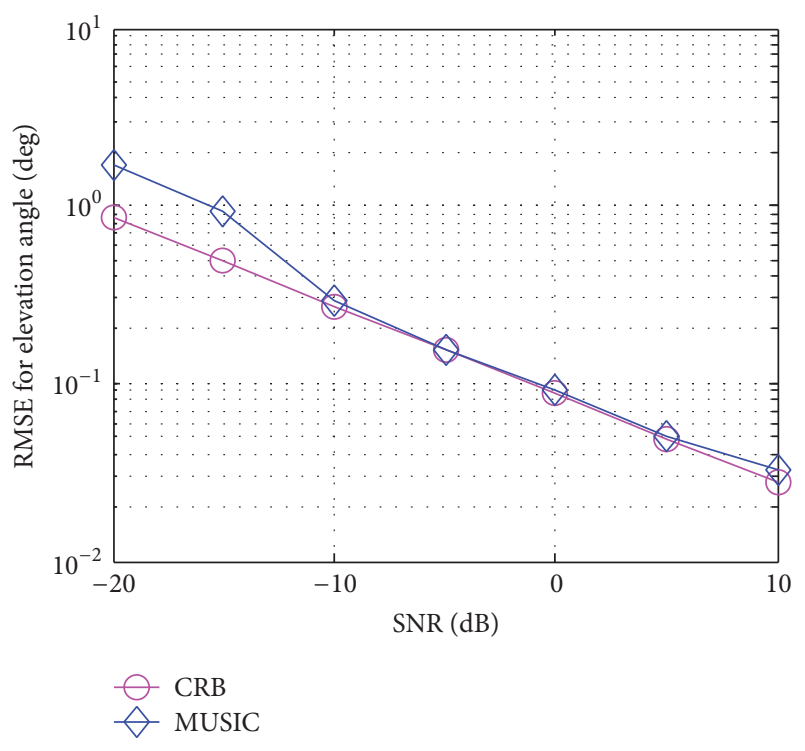

FIgURE 3: RMSE for elevation angle versus SNR.

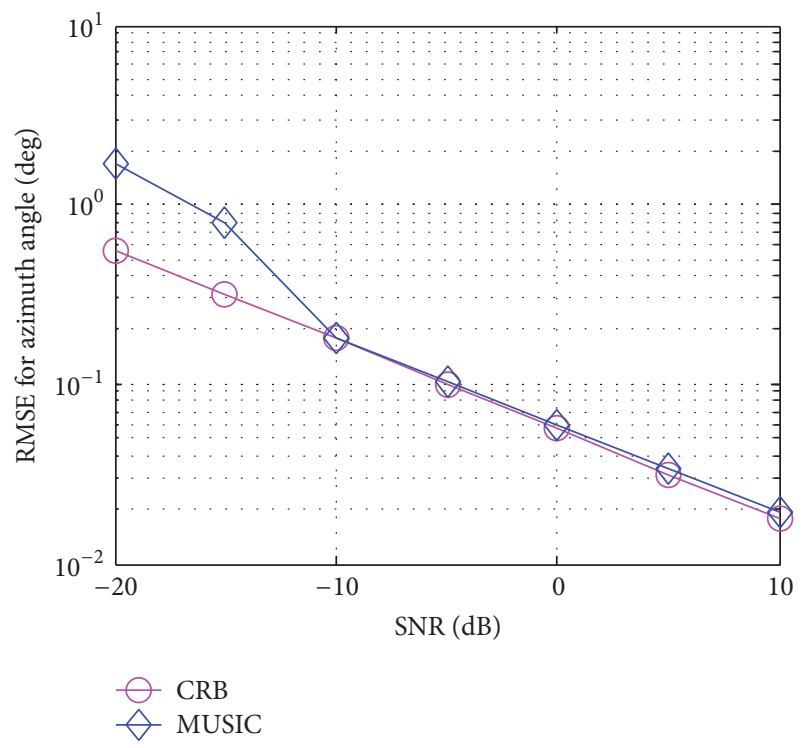

FIgURE 4: RMSE for azimuth angle versus SNR.

In order to analyze the influence of gain-phase error from transmit and receive antennas on target localization, we consider the case in which the gain-phase error exists in each antenna. Besides, the gain and phase errors are supposed to be Gaussian distributed random variables with zero mean and standard deviations $\sigma_{a}$ and $\sigma_{p}$, respectively. Figures 6 and 7 depict the RMSE versus the gain-phase errors of arrays under $\mathrm{SNR}=-10 \mathrm{~dB}$, where the plane curve below in each figure represents the corresponding $\mathrm{CRB}$ at $\mathrm{SNR}=-10 \mathrm{~dB}$. It can be seen from Figures 6 and 7 that the gain errors in array elements influence the target localization inconspicuously. The phase errors in array elements have a small impact on the target localization in the presence of small standard deviations $\sigma_{p}$. The RMSEs of the parameters estimation

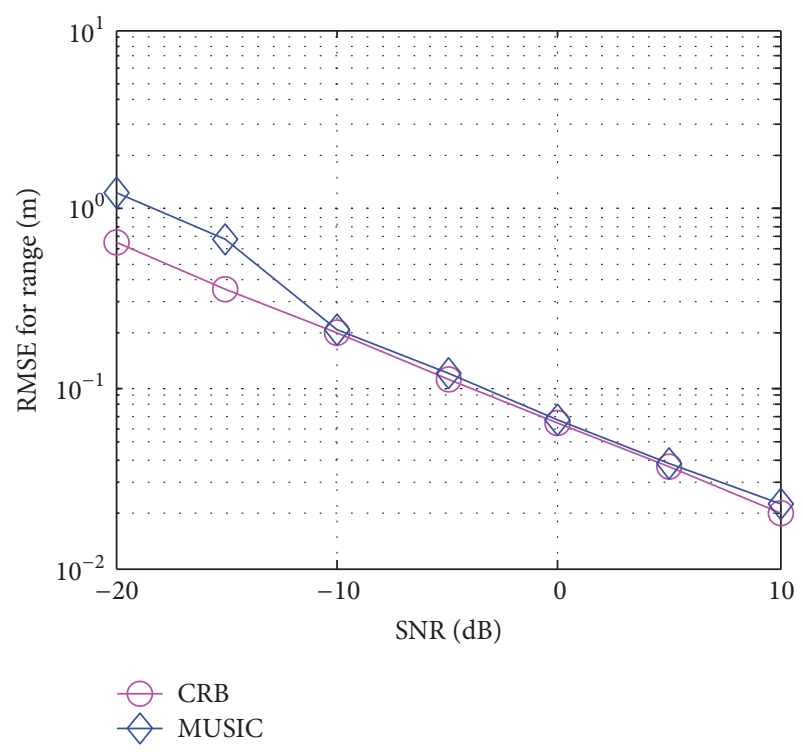

FIGURE 5: RMSE for range versus SNR.

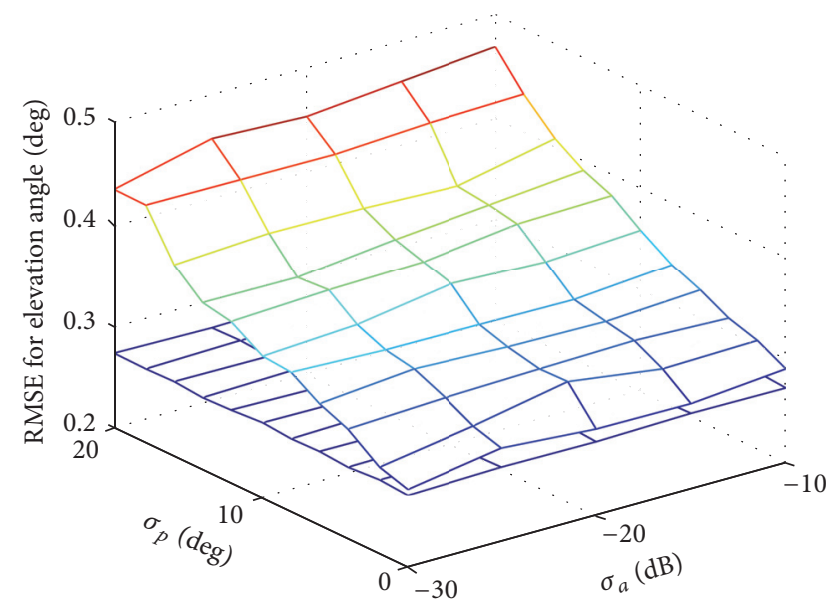

FIGURE 6: RMSE for elevation angle versus gain-phase errors.

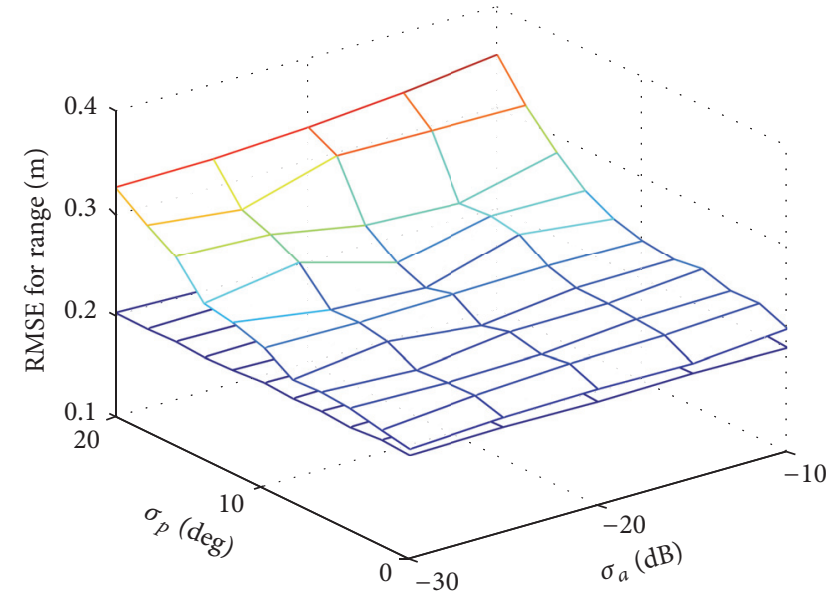

FIGURE 7: RMSE for range versus gain-phase errors. 


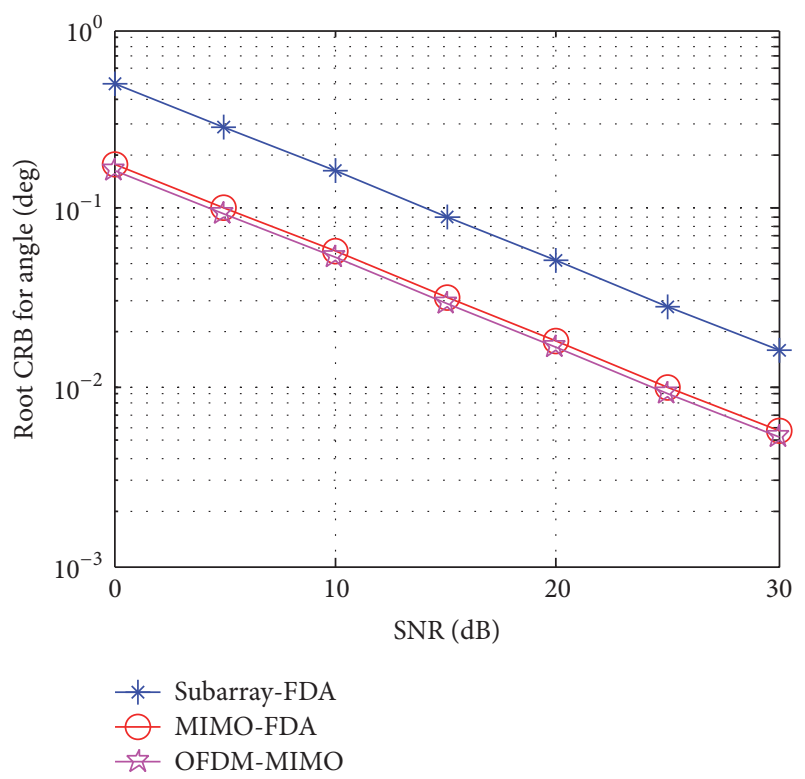

FIgURE 8: Root angle CRB versus SNR.

become larger as the phase errors further increase. However, the estimation precision is acceptable. In fact, the array model uncertainties are usually small. Therefore, our method has good robustness against the gain-phase errors of arrays.

5.2. Performance Comparison in Target Localization. In this section, our aim is to demonstrate the advantages of our OFDM-MIMO radar as compared to the MIMO-FDA radar [9] and the subarray-based FDA radar [10] in terms of CRBs for angle and range. It is worth noting that the comparison is under the uniform linear array (ULA) due to the literature $[9,10]$ using the ULA. We assume a target of interest is located at direction $\theta=10^{\circ}$ and range $r=15.105 \mathrm{~km}$. To be fair, consider a uniform linear array of $N=20$ antenna element for transmit and receive side, and the whole transmit array is divided into $P=2$ subarrays. It is worth highlighting that fully overlapped subarrays are employed in the MIMO-FDA radar. Besides, the frequency increment $\Delta f=B /(N-P)=$ $0.0556 \mathrm{MHz}$ is adopted for the MIMO-FDA radar and $\Delta f_{2}=$ $-\Delta f_{1}$ are selected for subarray-based FDA radar, where $\Delta f_{1}=$ $0.0556 \mathrm{MHz}$. Consider the target localization of the method in [9] which is based on a double-pulse; thus the CRBs of the other two radars are obtained based on two snapshots.

Figures 8 and 9 show the CRBs of three radars versus SNR. We can see that the angle CRBs of OFDM-MIMO and MIMO-FDA radar are approximately equivalent and better than subarray-based FDA radar, which can be attributed to the number of subarrays with unique waveforms at the transmitter and extended data vector at the receiver. Besides, OFDM-MIMO radar has lower range CRB as compared to the other two radars. The superiority of proposed OFDMMIMO radar in range estimation can be attributed to the larger frequency increment.

5.3. Impacts on CRBs. In this simulation, we conduct six experiments, say experiments $1,2,3,4,5$, and 6 , to analyze

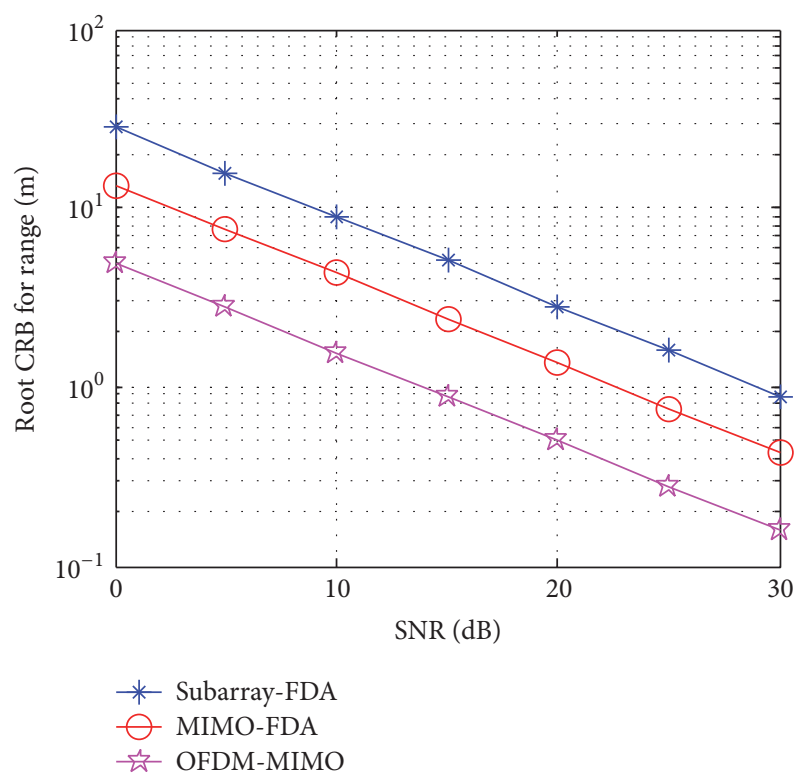

FIgURE 9: Root range CRB versus SNR.

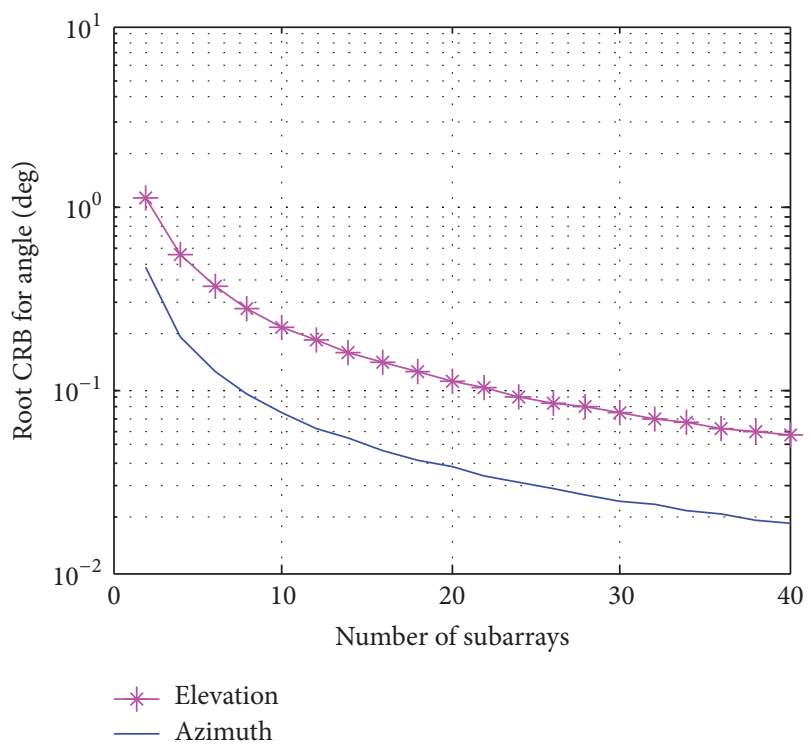

FIGURE 10: Root CRB for angle versus number of subarrays.

the CRBs in different conditions. For the sake of brevity, we did not show the CRBs for azimuth angle expect for experiments 1 and 5 since the characteristic of azimuth angle is similar to elevation angle.

5.3.1. Experiment 1: Comparison of CRBs with the Number of Subarrays. Consider $K_{y}=1$; therefore $K=K_{x}$, and the SNR is fixed at $0 \mathrm{~dB}$. The CRBs of angle and range versus the number of subarrays are plotted in Figures 10 and 11, respectively. It can be seen from Figures 10 and 11 that the CRBs decrease as the number of subarrays increases; in other words, more subarrays mean better performance, and this coincides with the qualitative conclusion (1) in Section 4.1. However, when the number of subarrays is more than 20 , 


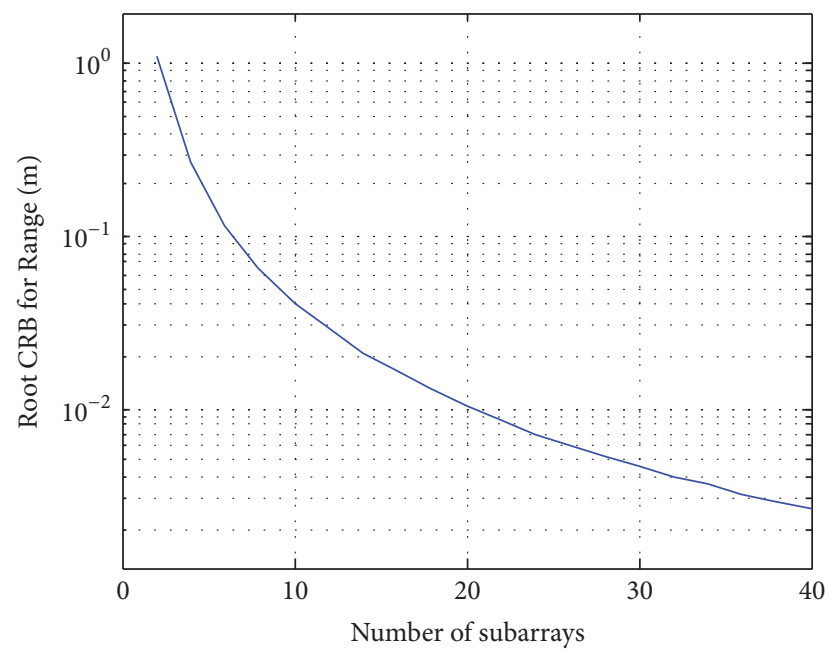

FIGURE 11: Root CRB for range versus number of subarrays.

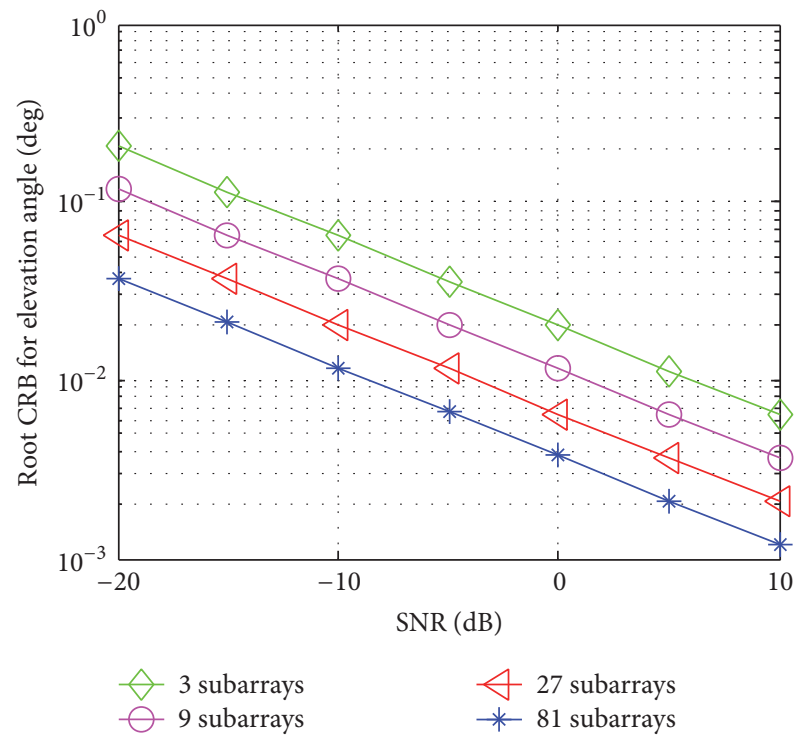

FIGURE 12: Root CRB for elevation angle versus SNR.

the improvement of CRBs performance is not evident if the number of the subarrays further increases.

5.3.2. Experiment 2: Comparison of CRBs with Different Subarrays Partition under the Same SNR. Figures 12 and 13 show the subarrays vary from 3 subarrays with 27 elements each in the first case to 81 subarrays with 1 element each in the last case. Since the virtual array of the system is larger with the increasing of the number of subarrays, the CRBs performance for angle is improved. Similarly, since the equivalent bandwidth of the echo is wider with the increasing of the number of subarrays, the CRB for range is lower. It is can be concluded from Figures 12 and 13 that the larger the number of the subarrays is divided the better estimation performance can be achieved. However, the computation complexity is higher at the same time.

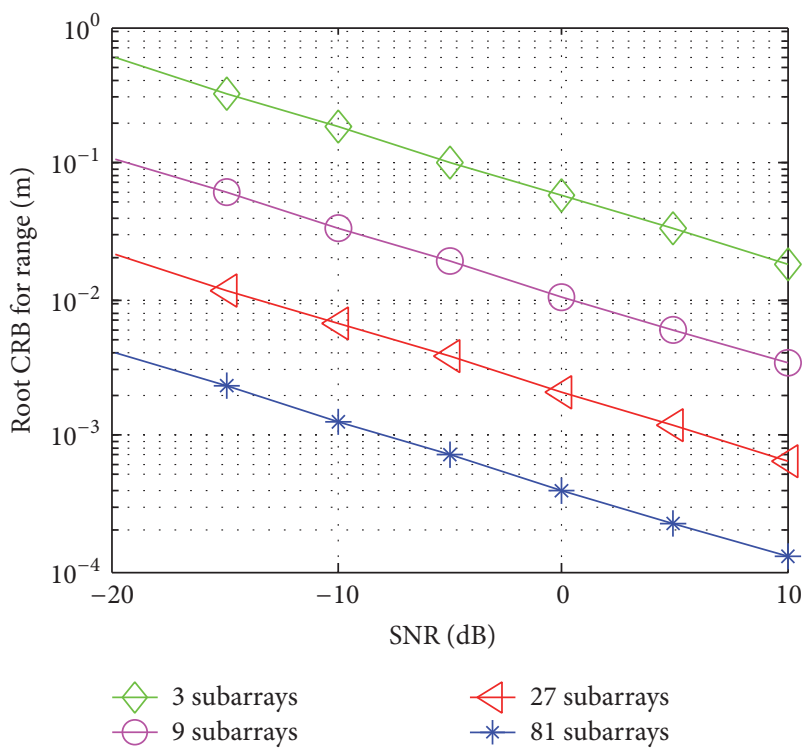

FIgURE 13: Root CRB for range versus SNR.

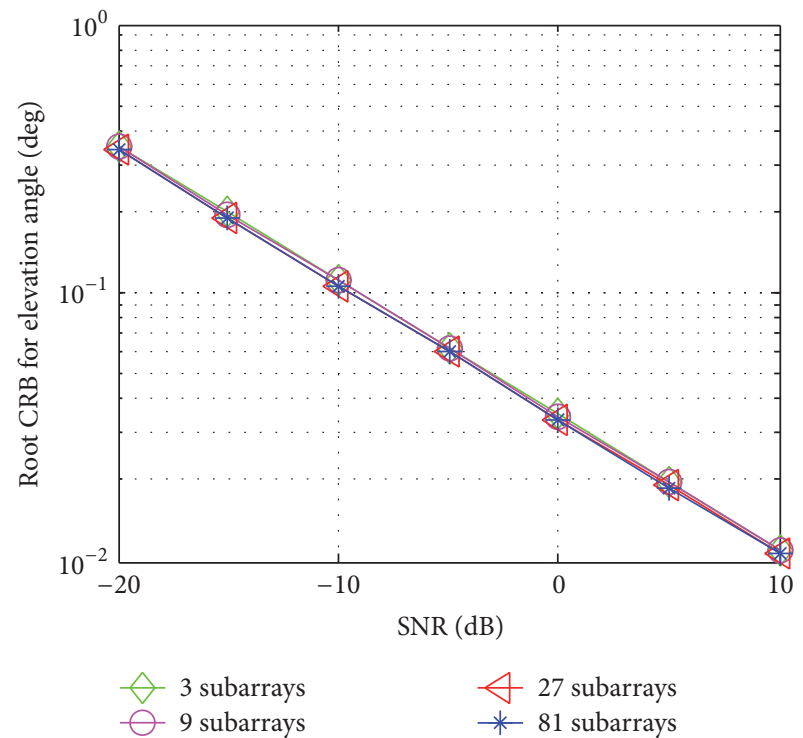

FIgURE 14: Root CRB for elevation angle versus SNR.

5.3.3. Experiment 3: Comparison of CRBs with Subarrays Partition under the Identical Total Transmitted Power. Assume that the subarray partition is similar to experiment 2, without loss of generality, we set the noise power $\sigma_{n}^{2}=1$ and $\alpha=1$. Figures 14 and 15 depict the CRBs of angle and range versus the SNR (i.e., total transmitted power) for different number of subarrays, respectively. Figure 14 illustrates that the CRB for angle is approximately equivalent to the increase of the number of subarrays which is different from experiment 2. Although more subarrays result in the increase of the virtual aperture, it means small coherent processing gain since the system makes a tradeoff between the effective aperture of virtual array and coherent gain. It can be observed from Figure 15 that the $\mathrm{CRB}$ performance for range is better as the 


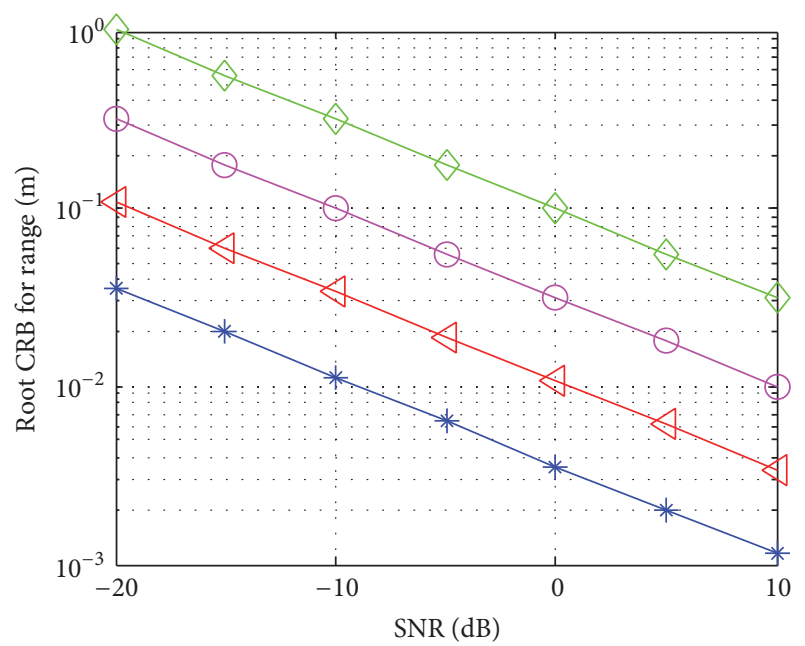

\begin{tabular}{ll}
$\forall 3$ subarrays & $\& 27$ subarrays \\
\hdashline s subarrays & $* 81$ subarrays
\end{tabular}

FIGURE 15: Root CRB for range versus SNR.

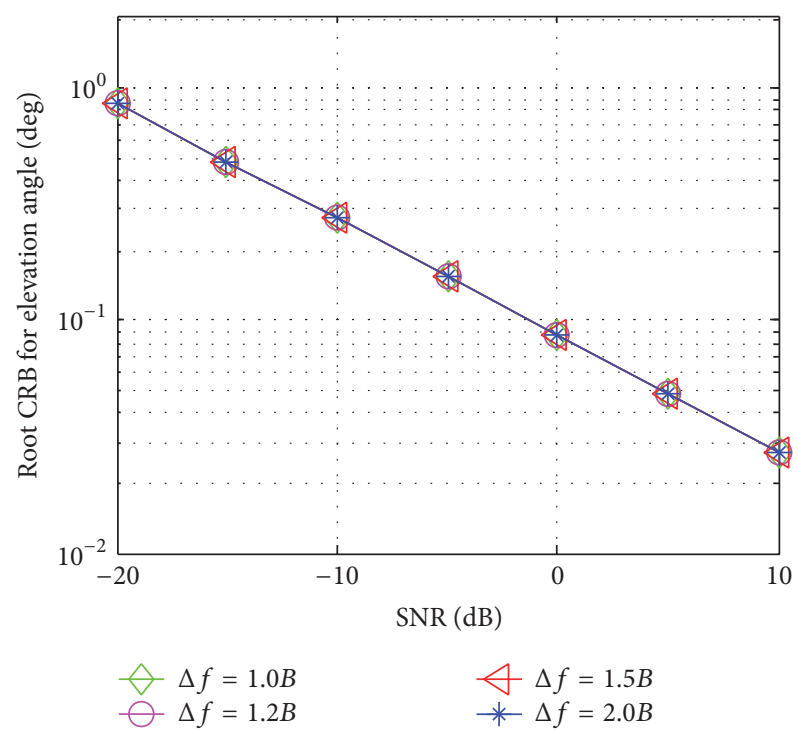

FIgURE 16: Root CRB for elevation angle versus frequency interval.

number of subarrays is larger since the equivalent bandwidth becomes wider.

5.3.4. Experiment 4: Comparison of CRBs with the Frequency Interval. In this experiment, the frequency interval varies from $1 B$ to $2 B$. Figures 16 and 17 show the CRBs of angle and range versus the SNR with different frequency interval, respectively. It is shown that the CRBs of the range decrease as the frequency interval increases since the system equivalent bandwidths become wider, which verified the correctness of the qualitative conclusions (2) in Section 4.1, while the CRBs for the angle are approximately equivalent as the effective array aperture has not increased with the change of frequency interval.

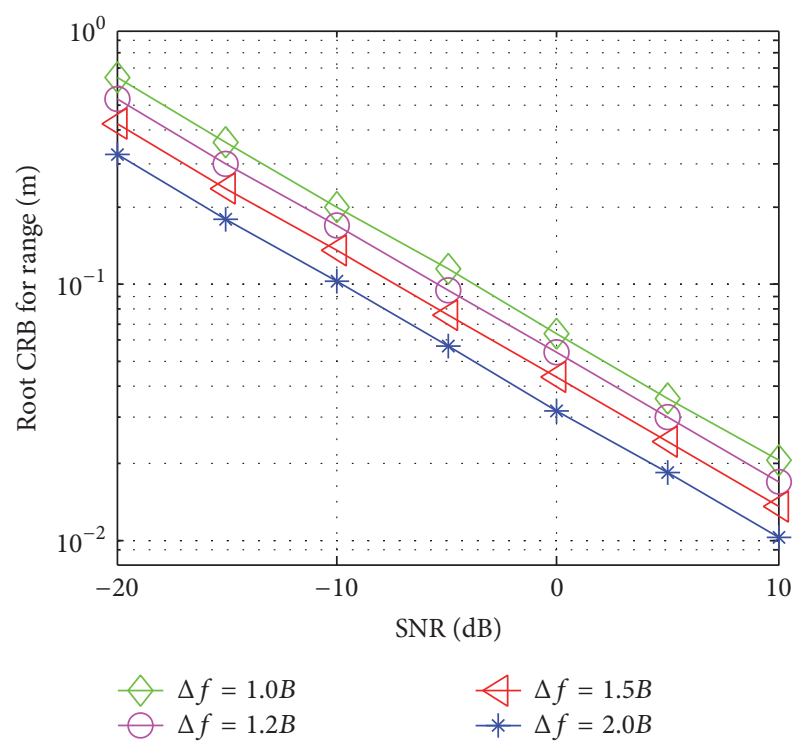

FIGURE 17: Root CRB for range versus frequency interval.

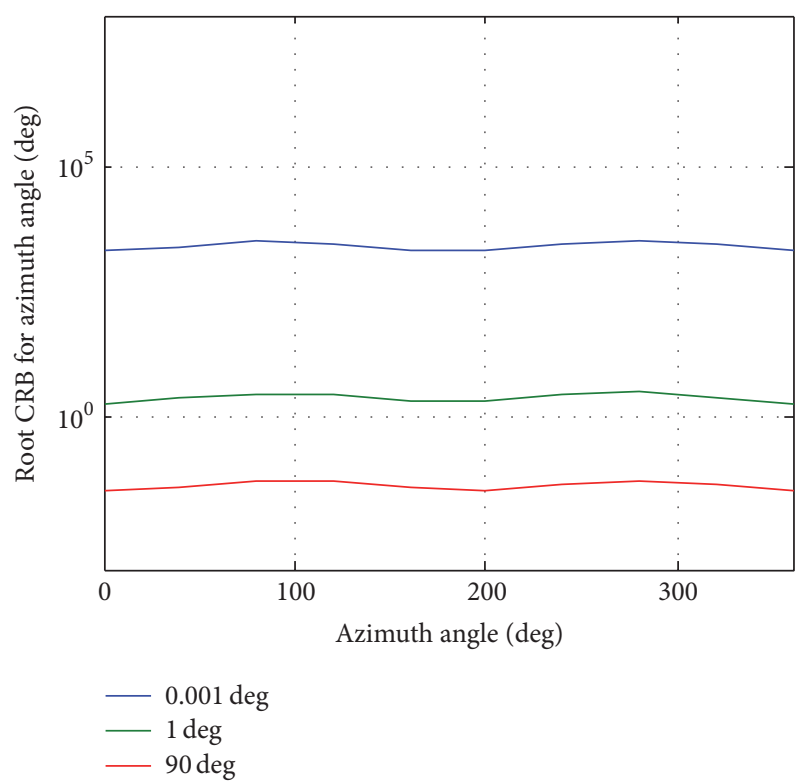

FIGURE 18: Root CRB for azimuth angle versus azimuth angle.

5.3.5. Experiment 5: Comparison of CRBs with the Angle and Range. The elevation angle of the target is set as $0.001^{\circ}, 1^{\circ}$, and $90^{\circ}$, respectively. Figures 18 and 19 illustrate the CRBs of azimuth angle and range versus the azimuth angle, respectively. It can be observed from Figure 18 that it has no significant difference as azimuth angle varies. Besides, when the elevation angle is close to $0^{\circ}$, the CRB increase rapidly, because the received target echo impinges on the array vertically; thus the array has no resolution capability in azimuth. Figures 20 and 21 show the CRBs of angle and range versus elevation angle when the azimuth angle of the target are set as $0^{\circ}$, $30^{\circ}$, and $90^{\circ}$, respectively. It can be observed from Figure 20 that the CRB of azimuth angle is relatively insensitive to 


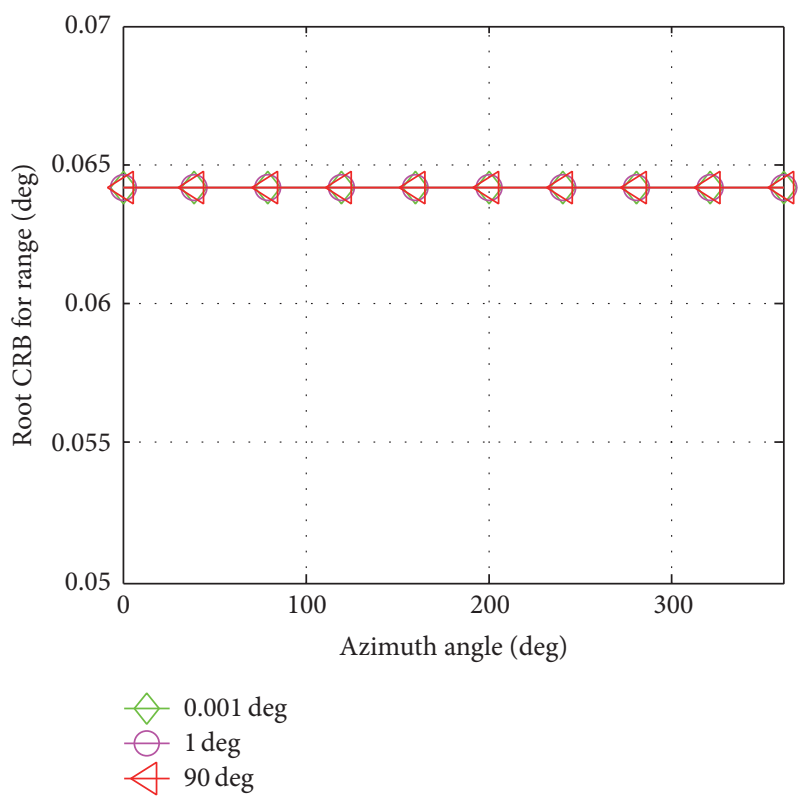

FIGURE 19: Root CRB for range versus the azimuth angle.

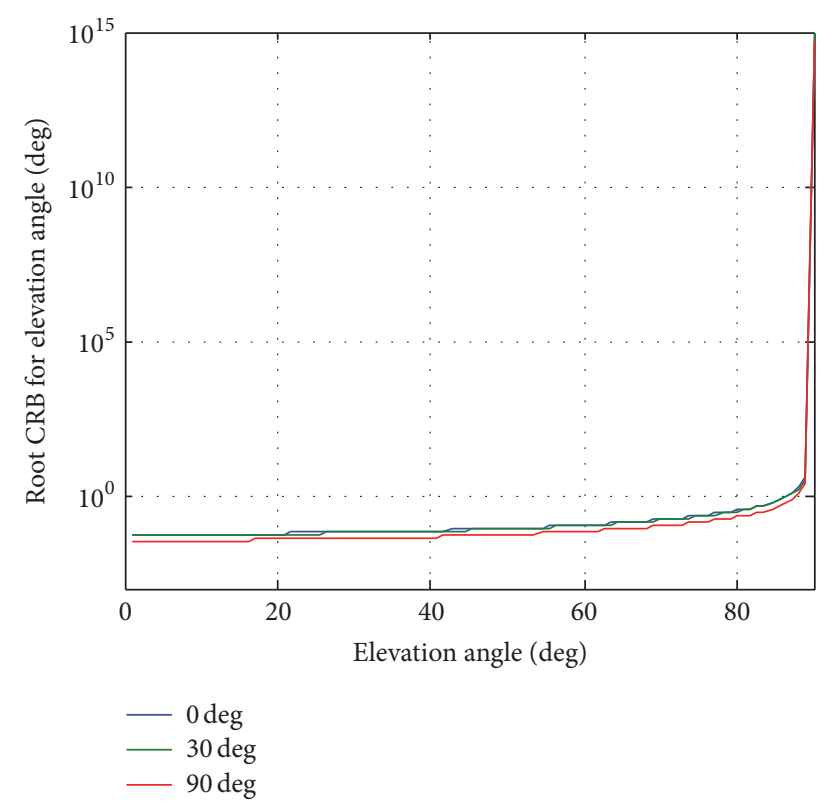

FIGURE 20: Root CRB for elevation angle versus elevation angle.

the variation of azimuth angle. When the elevation angle approaches $90^{\circ}$, the CRB is increasing dramatically, because the echo impinges parallel to the array; thus the array has no resolution capability in elevation. As we can see from Figures 19 and 21, the CRB for range is unaffected whether the azimuth angle varies from $0^{\circ}$ to $360^{\circ}$ or the elevation angle varies from $0^{\circ}$ to $90^{\circ}$.

5.3.6. Experiment 6: Comparison of CRBs with the Frequency Coding Sequence. In this experiment, four permutations of 7 integers are generated randomly,

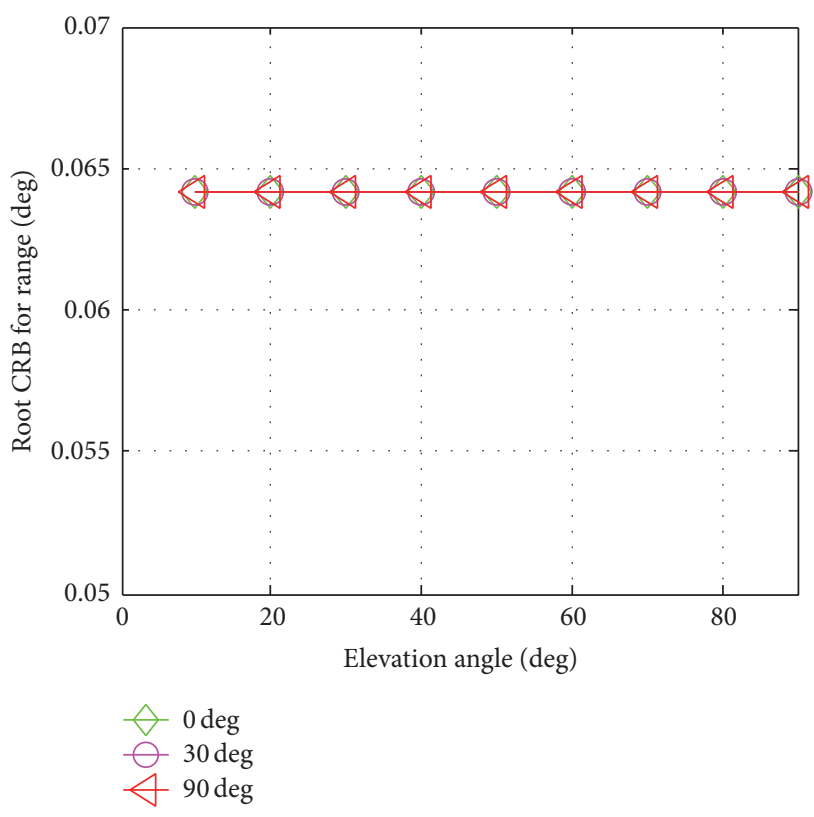

FIGURE 21: Root CRB for range versus the elevation angle.

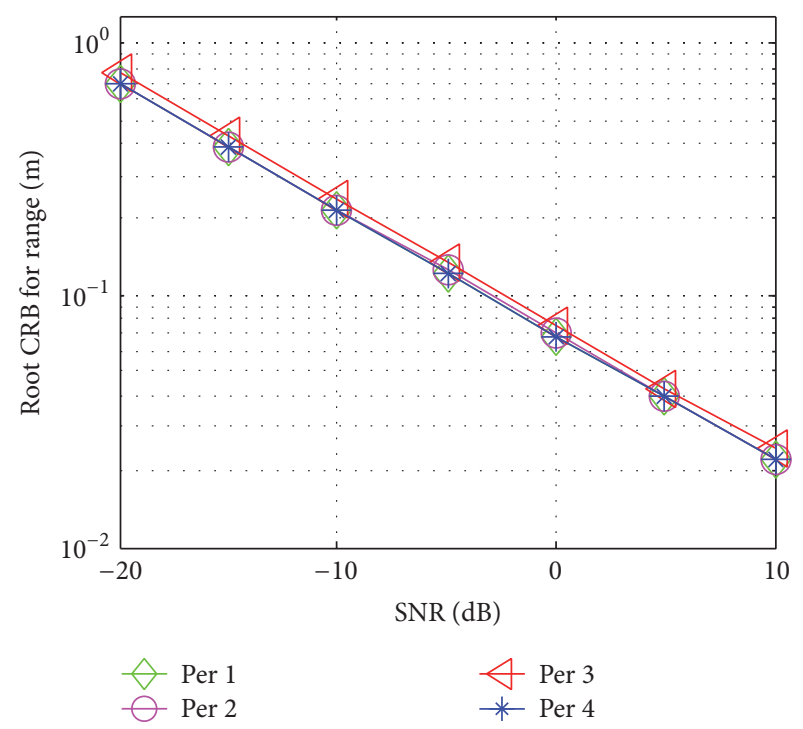

Figure 22: Root CRB for angle versus frequency coding sequence.

which are $\left[\begin{array}{llllllll}0 & 4 & 7 & 6 & 5 & 3 & 2 & 1\end{array}\right],\left[\begin{array}{llllllll}2 & 5 & 7 & 3 & 1 & 4 & 0 & 6\end{array}\right]$, $\left[\begin{array}{llllllll}3 & 5 & 1 & 6 & 0 & 7 & 2 & 4\end{array}\right]$, and $\left[\begin{array}{llllllll}0 & 7 & 6 & 1 & 2 & 4 & 5 & 3\end{array}\right]$, respectively. Figures 22 and 23 show the CRBs of angle and range versus the SNR with different frequency coding sequence, respectively. It can be seen that the CRBs of the angle and range are affected by the frequency coding sequence. In other words, different frequency coding sequences result in different CRBs of the angle and range, which offers a way to choose appropriate frequency coding sequence for improved performance of target localization.

5.4. CRB Optimization. In the last simulation, we conduct experiment to validate the effectiveness of the proposed CRB 


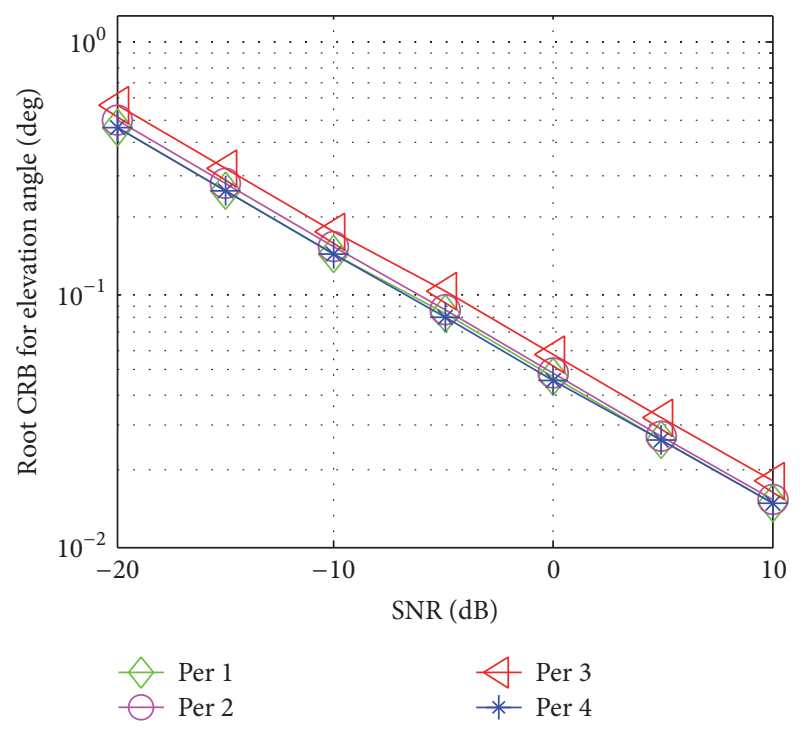

FIGURE 23: Root CRB for range versus frequency coding sequence.

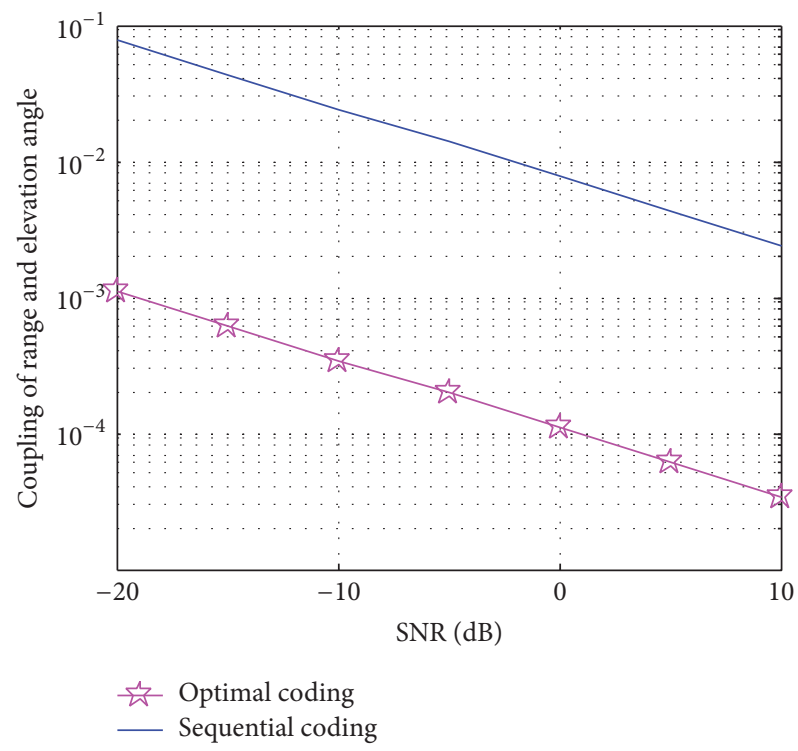

FIGURE 24: Coupling of elevation angel and range.

optimization proposal. Other parameters are the same as the first simulation. The optimized transmit frequency coding sequence $\mathbf{k}=\left[\begin{array}{llllllll}7 & 2 & 4 & 1 & 0 & 5 & 3 & 6\end{array}\right]$ is adopted. The absolute value of the coupling between elevation angle and range is shown in Figure 24. It is seen that the effect of the coupling between elevation angel and range is mitigated obviously after the frequency coding optimization. Thus the CRBs for angle and range reduced evidently which can be observed from Figures 25 and 26, respectively.

\section{Conclusion}

In this paper, a $3 \mathrm{D}$ target localization method is presented for the proposed OFDM-MIMO radar, and the superresolution performance in range is achieved by utilizing the additional

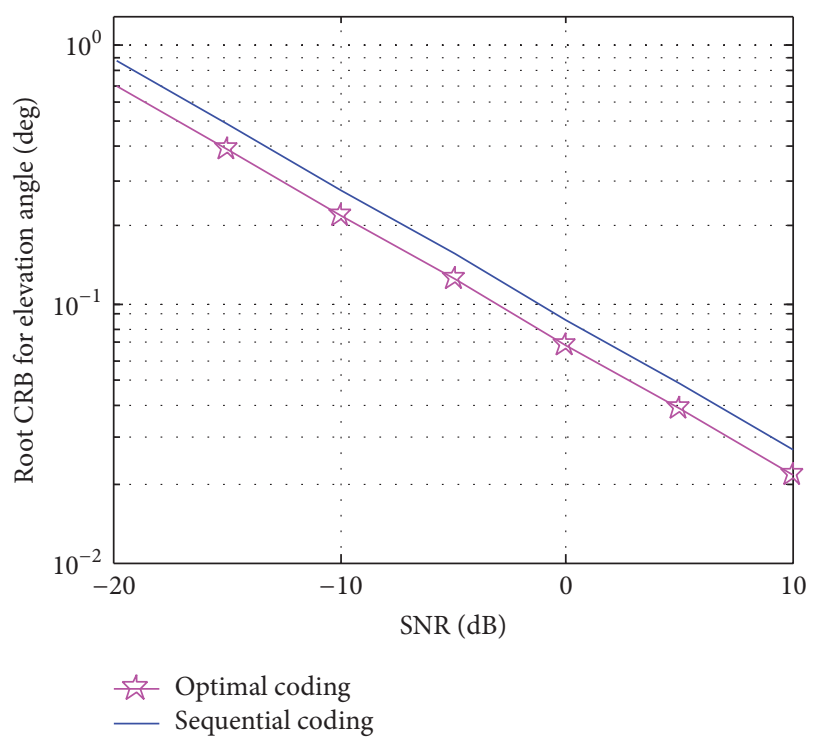

FIGURE 25: Root CRB for elevation angle versus SNR.

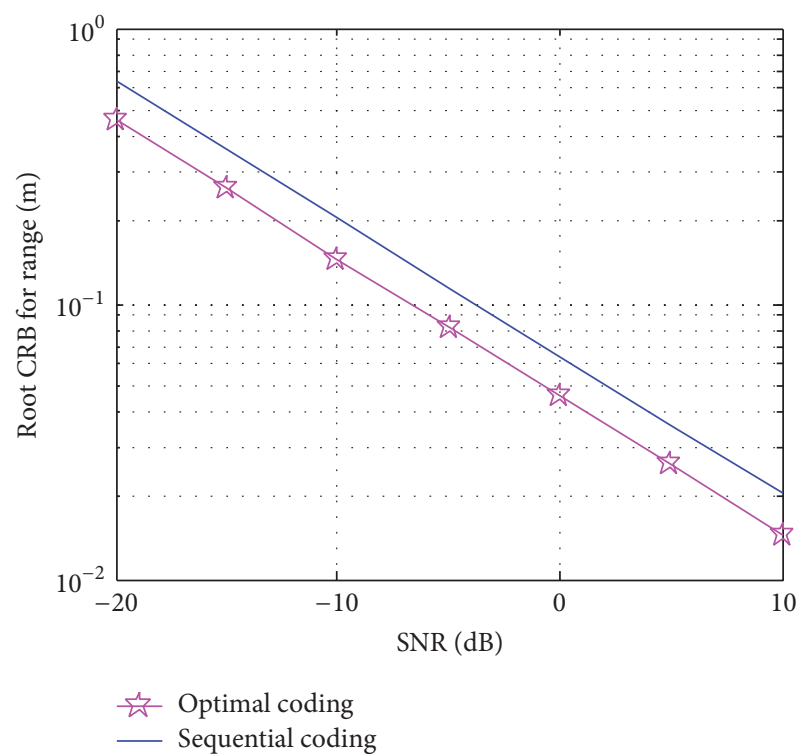

FIgURE 26: Root CRB for range versus SNR.

DOFs in range. Moreover, the corresponding CRBs for the joint range and angle are derived and a CRB optimum proposal based on frequency coding is proposed; it is worth noting that the criterion of (35) can be also used to optimize subarray partition so as to improve CRB performance. Additionally, the superior performance in range domain of the proposed system and approach are verified by extensive simulation results. Potential future work includes optimization of subarray segmentation to minimize the CRB for range and angle estimation, and adaptive beamforming in both angle and range with better interference suppression capability; thus the detection performance of target will be improved by employing the proposed system. We believe that applying the technology of frequency diversity to MIMO is a promising 
concept that will play an enormously important role in the future radar.

\section{Appendix}

For clarity, we write matrix $\mathbf{J}$ as

$$
\mathbf{J}=\left[\begin{array}{ll}
\mathbf{J}_{11} & \mathbf{J}_{12} \\
\mathbf{J}_{12}^{T} & \mathbf{J}_{22}
\end{array}\right]
$$

where

$$
\begin{aligned}
& \mathbf{J}_{11}=\left[\begin{array}{lll}
J_{\theta \theta} & J_{\theta \varphi} & J_{\theta r} \\
J_{\theta \varphi} & J_{\varphi \varphi} & J_{\varphi r} \\
J_{\theta r} & J_{\varphi r} & J_{r r}
\end{array}\right] \\
& \mathbf{J}_{12}=\left[\begin{array}{ll}
J_{\theta \beta_{R}} & J_{\theta \beta_{I}} \\
J_{\varphi \beta_{R}} & J_{\varphi \beta_{I}} \\
J_{r \beta_{R}} & J_{r \beta_{I}}
\end{array}\right] \\
& \mathbf{J}_{22}=\left[\begin{array}{ll}
J_{\beta_{R} \beta_{R}} & J_{\beta_{R} \beta_{I}} \\
J_{\beta_{R} \beta_{I}} & J_{\beta_{I} \beta_{I}}
\end{array}\right] .
\end{aligned}
$$

For the sake of brevity, we sign $\boldsymbol{\mu}=\boldsymbol{\mu}(\psi)$. Thus, the FIM can be derived as
$\mathbf{J}_{11}$

$$
=\frac{2 L|\beta|^{2}}{\sigma_{n}^{2}}\left[\begin{array}{ccc}
\left\|\boldsymbol{\mu}_{\theta}\right\|^{2} & \operatorname{Re}\left\{\boldsymbol{\mu}_{\theta}^{H} \boldsymbol{\mu}_{\varphi}\right\} & \operatorname{Re}\left\{\boldsymbol{\mu}_{\theta}^{H} \boldsymbol{\mu}_{r}\right\} \\
\operatorname{Re}\left\{\boldsymbol{\mu}_{\theta}^{H} \boldsymbol{\mu}_{\varphi}\right\} & \left\|\boldsymbol{\mu}_{\varphi}\right\|^{2} & \operatorname{Re}\left\{\boldsymbol{\mu}_{\varphi}^{H} \boldsymbol{\mu}_{r}\right\} \\
\operatorname{Re}\left\{\boldsymbol{\mu}_{\theta}^{H} \boldsymbol{\mu}_{r}\right\} & \operatorname{Re}\left\{\boldsymbol{\mu}_{\varphi}^{H} \boldsymbol{\mu}_{r}\right\} & \left\|\boldsymbol{\mu}_{r}\right\|^{2}
\end{array}\right]
$$$$
\mathbf{J}_{12}=\frac{2 L}{\sigma_{n}^{2}}\left[\begin{array}{ll}
\operatorname{Re}\left\{\boldsymbol{\mu}_{\theta}^{H} \boldsymbol{\mu} \beta^{*}\right\} & -\operatorname{Im}\left\{\boldsymbol{\mu}_{\theta}^{H} \boldsymbol{\mu} \beta^{*}\right\} \\
\operatorname{Re}\left\{\boldsymbol{\mu}_{\varphi}^{H} \boldsymbol{\mu} \beta^{*}\right\} & -\operatorname{Im}\left\{\boldsymbol{\mu}_{\varphi}^{H} \boldsymbol{\mu} \beta^{*}\right\} \\
\operatorname{Re}\left\{\boldsymbol{\mu}_{r}^{H} \boldsymbol{\mu} \beta^{*}\right\} & -\operatorname{Im}\left\{\boldsymbol{\mu}_{r}^{H} \boldsymbol{\mu} \beta^{*}\right\}
\end{array}\right]
$$

$$
\mathbf{J}_{22}=\frac{2 L\|\mu\|^{2}}{\sigma_{n}^{2}} \mathbf{I}_{2}
$$

According to the matrix inversion lemma, the inverse matrix of $\mathbf{J}$ is

$$
\begin{aligned}
& \mathbf{J}^{-1} \\
& =\left[\begin{array}{cc}
\left(\mathbf{J}_{11}-\mathbf{J}_{12} \mathbf{J}_{22}^{-1} \mathbf{J}_{12}^{T}\right)^{-1} & -\mathbf{J}_{11}^{-1} \mathbf{J}_{12}\left(\mathbf{J}_{22}-\mathbf{J}_{21} \mathbf{J}_{11}^{-1} \mathbf{J}_{12}\right)^{-1} \\
-\mathbf{J}_{22}^{-1} \mathbf{J}_{12}^{T}\left(\mathbf{J}_{11}-\mathbf{J}_{12} \mathbf{J}_{22}^{-1} \mathbf{J}_{12}^{T}\right)^{-1} & \left(\mathbf{J}_{22}-\mathbf{J}_{12}^{T} \mathbf{J}_{11}^{-1} \mathbf{J}_{12}\right)^{-1}
\end{array}\right],
\end{aligned}
$$

where submatrix $\left(\mathbf{J}_{11}-\mathbf{J}_{12} \mathbf{J}_{22}^{-1} \mathbf{J}_{12}^{T}\right)^{-1}$ corresponds to the CRB of the parameters $(\theta, \varphi, r)$ which are of interest. After some matrix manipulations, we obtain

$$
\begin{aligned}
& \mathbf{Q}=\left(\mathbf{J}_{11}-\mathbf{J}_{12} \mathbf{J}_{22}^{-1} \mathbf{J}_{12}^{T}\right) \\
& =2 L S N R\left[\begin{array}{ccc}
\left\|\boldsymbol{\mu}_{\theta}\right\|^{2}-\frac{\mid \boldsymbol{\mu}_{\theta}^{H} \boldsymbol{\mu}^{2}}{\|\boldsymbol{\mu}\|^{2}} & \operatorname{Re}\left\{\boldsymbol{\mu}_{\theta}^{H} \boldsymbol{\mu}_{\varphi}\right\}-\frac{\operatorname{Re}\left\{\boldsymbol{\mu}^{H} \boldsymbol{\mu}_{\theta} \boldsymbol{\mu}_{\varphi}^{H} \boldsymbol{\mu}\right\}}{\|\boldsymbol{\mu}\|^{2}} & \operatorname{Re}\left\{\boldsymbol{\mu}_{\theta}^{H} \boldsymbol{\mu}_{r}\right\}-\frac{\operatorname{Re}\left\{\boldsymbol{\mu}^{H} \boldsymbol{\mu}_{\theta} \boldsymbol{\mu}_{r}^{H} \boldsymbol{\mu}\right\}}{\|\boldsymbol{\mu}\|^{2}} \\
\operatorname{Re}\left\{\boldsymbol{\mu}_{\theta}^{H} \boldsymbol{\mu}_{\varphi}\right\}-\frac{\operatorname{Re}\left\{\boldsymbol{\mu}^{H} \boldsymbol{\mu}_{\theta} \boldsymbol{\mu}_{\varphi}^{H} \boldsymbol{\mu}\right\}}{\|\boldsymbol{\mu}\|^{2}} & \left\|\boldsymbol{\mu}_{\varphi}\right\|^{2}-\frac{\left|\boldsymbol{\mu}_{\varphi}^{H} \boldsymbol{\mu}\right|^{2}}{\|\boldsymbol{\mu}\|^{2}} & \operatorname{Re}\left\{\boldsymbol{\mu}_{\varphi}^{H} \boldsymbol{\mu}_{r}\right\}-\frac{\operatorname{Re}\left\{\boldsymbol{\mu}^{H} \boldsymbol{\mu}_{\varphi} \boldsymbol{\mu}_{r}^{H} \boldsymbol{\mu}\right\}}{\|\boldsymbol{\mu}\|^{2}} \\
\operatorname{Re}\left\{\boldsymbol{\mu}_{\theta}^{H} \boldsymbol{\mu}_{r}\right\}-\frac{\operatorname{Re}\left\{\boldsymbol{\mu}^{H} \boldsymbol{\mu}_{\theta} \boldsymbol{\mu}_{r}^{H} \boldsymbol{\mu}\right\}}{\|\boldsymbol{\mu}\|^{2}} & \operatorname{Re}\left\{\boldsymbol{\mu}_{\varphi}^{H} \boldsymbol{\mu}_{r}\right\}-\frac{\operatorname{Re}\left\{\boldsymbol{\mu}^{H} \boldsymbol{\mu}_{\varphi} \boldsymbol{\mu}_{r}^{H} \boldsymbol{\mu}\right\}}{\|\boldsymbol{\mu}\|^{2}} & \left\|\boldsymbol{\mu}_{r}\right\|^{2}-\frac{\left|\boldsymbol{\mu}_{r}^{H} \boldsymbol{\mu}\right|^{2}}{\|\boldsymbol{\mu}\|^{2}}
\end{array}\right],
\end{aligned}
$$

where SNR $=|\beta|^{2} / \sigma_{n}^{2}$. Setting $\mathbf{Q}=2 L$ SNRQ $_{0}$, the inverse matrix of $\mathbf{Q}_{0}$ can be expressed as

$$
\mathbf{Q}_{0}^{-1}=\frac{\mathbf{Q}_{0}^{\dagger}}{\operatorname{det}\left(\mathbf{Q}_{0}\right)},
$$

where $\mathbf{Q}_{0}{ }^{\dagger}$ denotes the adjoint matrix of $\mathbf{Q}_{0}$. $\operatorname{det}(\cdot)$ is the determinant operator.

$$
\left[D_{\theta}, D_{\varphi}, D_{r}\right]^{T}=\frac{1}{2 L S N R} \operatorname{diag}\left(\mathbf{Q}_{0}^{-1}\right) .
$$

\section{Competing Interests}

The authors declare that there is no conflict of interests regarding the publication of this paper.

\section{Acknowledgments}

This work is supported in part by the National Natural Science Foundation of China under Grant nos. 61179015 and 61401503 , respectively.

\section{References}

[1] V. Ravenni and G. Pizziol, "Frequency diversity radar system: design, analysis and performances," in Proceedings of the $3 \mathrm{rd}$ European Radar Conference (EuRAD '06), pp. 221-224, IEEE, Manchester, UK, September 2006.

[2] V. Ravenni, "Performance evaluations of frequency diversity radar system," in Proceedings of the 4th European Radar 
Conference (EURAD '07), pp. 436-439, IEEE, Munich, Germany, October 2007.

[3] S. Sen and A. Nehorai, "OFDM MIMO radar with mutualinformation waveform design for low-grazing angle tracking," IEEE Transactions on Signal Processing, vol. 58, no. 6, pp. 31523162, 2010.

[4] P. F. Sammartino, C. J. Baker, and H. D. Griffiths, "Frequency diverse MIMO techniques for radar," IEEE Transactions on Aerospace and Electronic Systems, vol. 49, no. 1, pp. 201-222, 2013.

[5] W.-Q. Wang, "Phased-MIMO radar with frequency diversity for range-dependent beamforming," IEEE Sensors Journal, vol. 13, no. 4, pp. 1320-1328, 2013.

[6] J. W. Xu, G. S. Liao, S. H. Zhu, L. Huang, and H. C. So, "Joint range and angle estimation using MIMO radar with frequency diverse array," IEEE Transactions on Signal Processing, vol. 63, no. 13, pp. 3396-3410, 2015.

[7] J. Xu, G. Liao, S. Zhu, and H. C. So, "Deceptive jamming suppression with frequency diverse MIMO radar," Signal Processing, vol. 113, pp. 9-17, 2015.

[8] W.-Q. Wang, "Range-angle dependent transmit beampattern synthesis for linear frequency diverse arrays," IEEE Transactions on Antennas and Propagation, vol. 61, no. 8, pp. 4073-4081, 2013.

[9] W. Khan, I. M. Qureshi, A. Basit, and M. Zubair, "A double pulse MIMO frequency diverse array radar for improved range-angle localization of target," Wireless Personal Communications, vol. 82, no. 4, pp. 2199-2213, 2015.

[10] W.-Q. Wang, "Subarray-based frequency diverse array radar for target range-angle estimation," IEEE Transactions on Aerospace and Electronic Systems, vol. 50, no. 4, pp. 3057-3067, 2014.

[11] Y. Wang, W.-Q. Wang, and H. Shao, "Frequency diverse array radar cramér-rao lower bounds for estimating direction, range, and velocity," International Journal of Antennas and Propagation, vol. 2014, Article ID 830869, 15 pages, 2014.

[12] D. R. Fuhrmann, J. P. Browning, and M. Rangaswamy, "Signaling strategies for the hybrid MIMO phased-array radar," IEEE Journal on Selected Topics in Signal Processing, vol. 4, no. 1, pp. 66-78, 2010.

[13] W.-Q. Wang, "Space-time coding MIMO-OFDM SAR for highresolution imaging," IEEE Transactions on Geoscience and Remote Sensing, vol. 49, no. 8, pp. 3094-3104, 2011.

[14] R. Adve, R. Schneible, and R. McMillan, "Adaptive space/frequency processing for distributed aperture radars," in Proceedings of the IEEE Radar Conference, pp. 160-164, IEEE, Huntsville, Ala, USA, May 2003.

[15] X. Li, Z. Zhang, W. Mao, X. Wang, J. Lu, and W. Wang, "A study of frequency diversity MIMO radar beamforming," in Proceedings of the IEEE 10th International Conference on Signal Processing (ICSP '10), pp. 352-356, Beijing, China, October 2010.

[16] W.-Q. Wang and H. C. So, "Transmit subaperturing for range and angle estimation in frequency diverse array radar," IEEE Transactions on Signal Processing, vol. 62, no. 8, pp. 2000-2011, 2014.

[17] A. Hassanien and S. A. Vorobyov, "Phased-MIMO radar: a tradeoff between phased-array and MIMO radars," IEEE Transactions on Signal Processing, vol. 58, no. 6, pp. 3137-3151, 2010.

[18] M. A. Richards, Fundamentals of Radar Signal Processing, Tata McGraw-Hill Education, New York, NY, USA, 2005.

[19] Y. Zhang and B. P. Ng, "MUSIC-like DOA estimation without estimating the number of sources," IEEE Transactions on Signal Processing, vol. 58, no. 3, pp. 1668-1676, 2010.
[20] H. L. Van Trees, Detection, Estimation, and Modulation Theory III, John Wiley \& Sons, New York, NY, USA, 2001. 


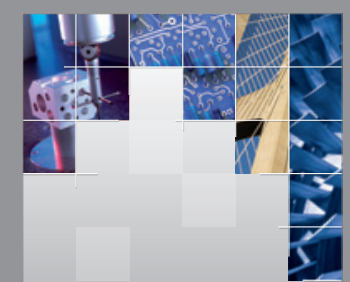

\section{Enfincering}
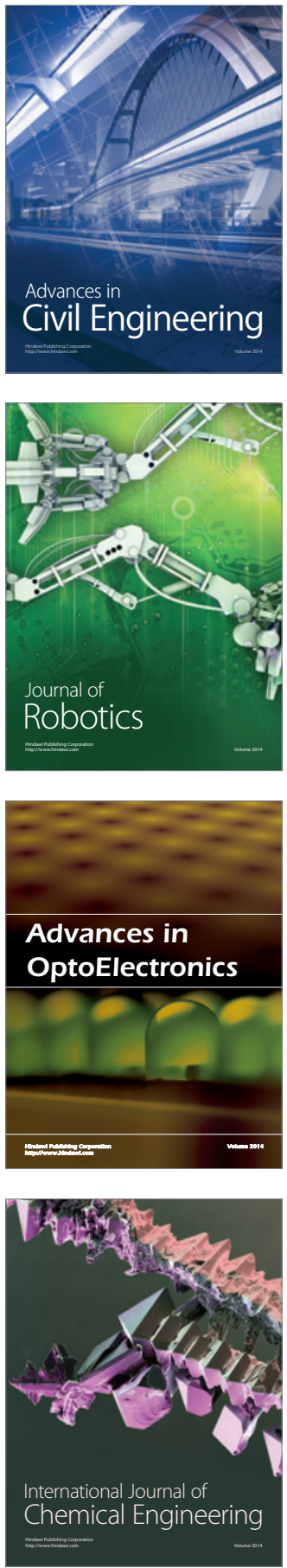

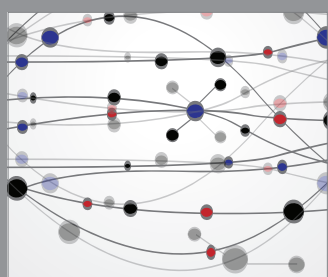

The Scientific World Journal

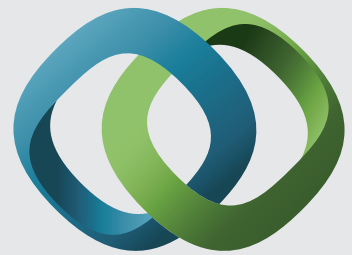

\section{Hindawi}

Submit your manuscripts at

https://www.hindawi.com
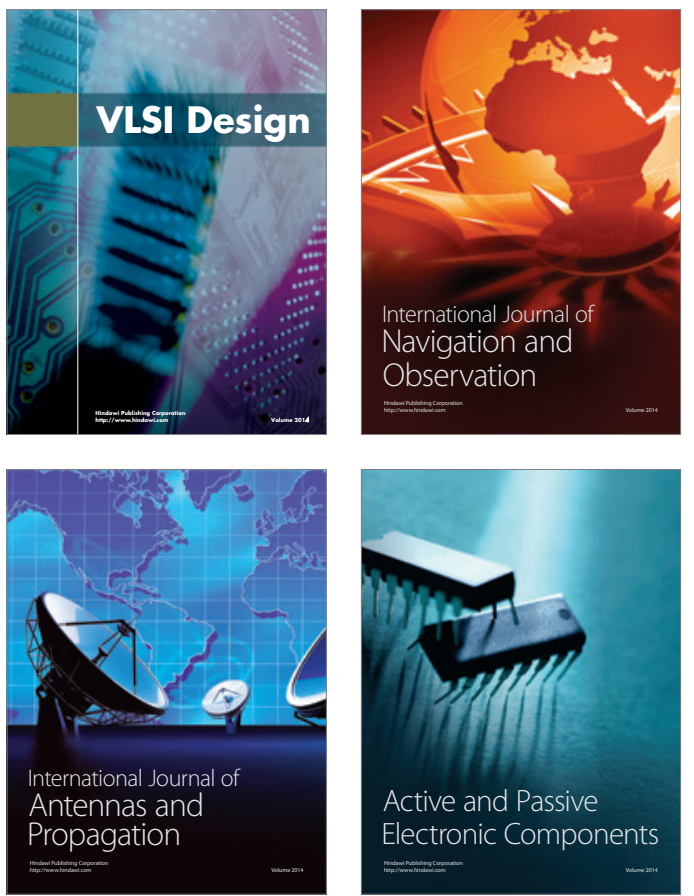
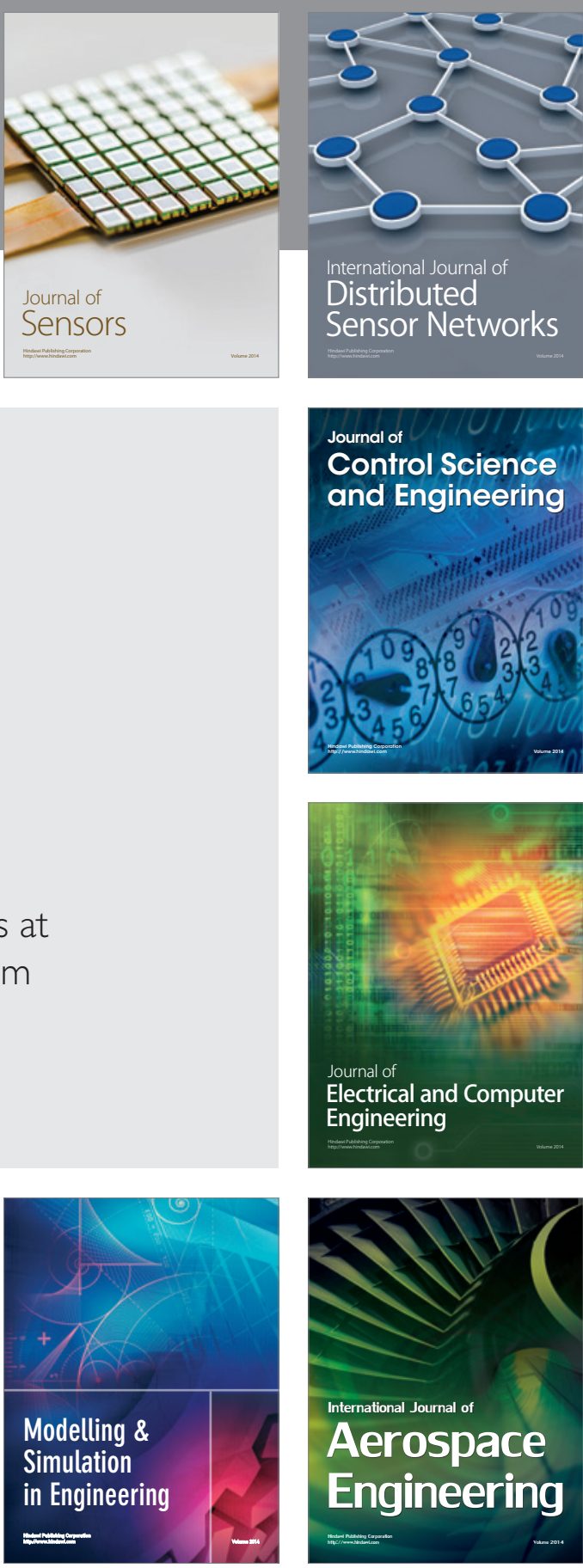

International Journal of

Distributed

Sensor Networks

$-$

Joumal of

Control Science

and Engineering
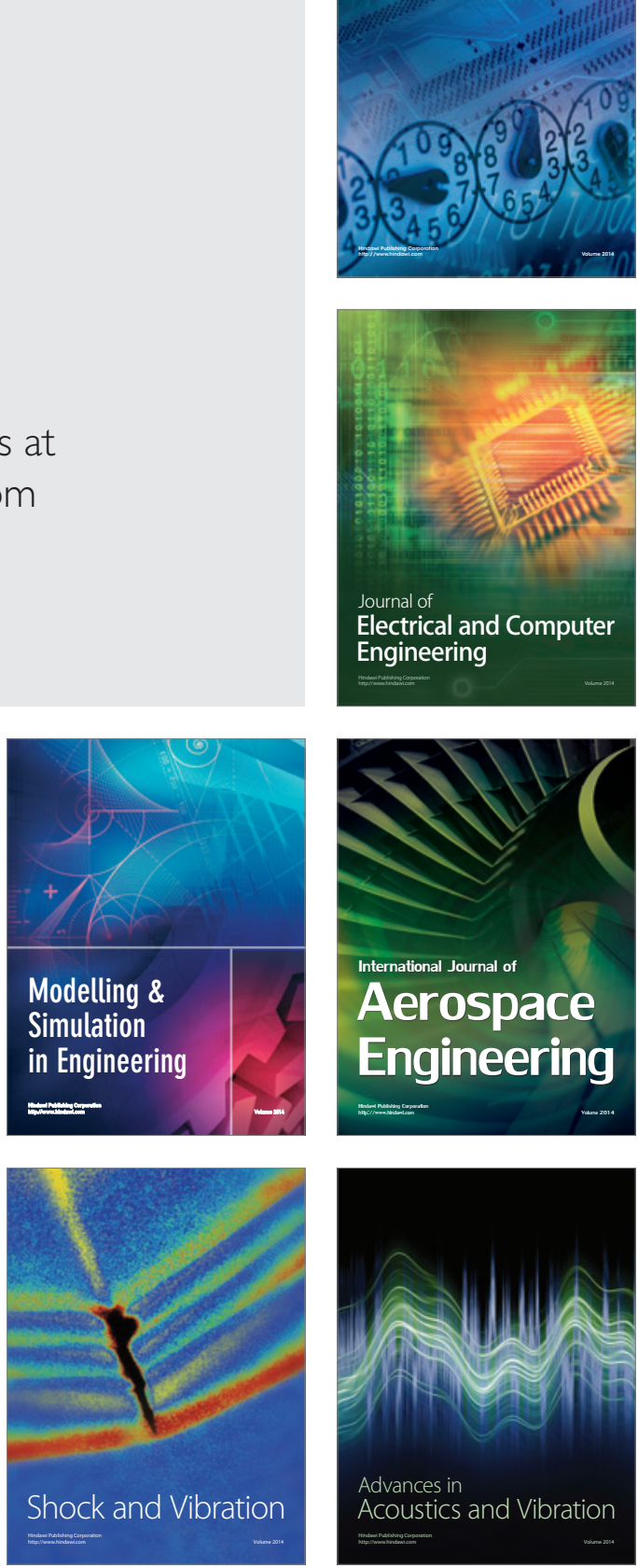